\title{
Metabolite discovery through global annotation of untargeted metabolomics data
}

2 Li Chen ${ }^{1,2}$, Wenyun Lu ${ }^{2,3}$, Lin Wang ${ }^{2,3}$, Xi Xing ${ }^{2,3}$, Ziyang Chen ${ }^{1,4}$, Xin Teng ${ }^{2}$, Xianfeng Zeng ${ }^{2,3}$,

3 Antonio D.Muscarella2, Yihui Shen², Alexis Cowan ${ }^{2,4}$, Melanie R. McReynolds ${ }^{2,3}$, Brandon

4 Kennedy ${ }^{5}$, Ashley M. Lato ${ }^{6}$, Shawn R. Campagna ${ }^{6}$, Mona Singh ${ }^{2,7}$, Joshua Rabinowitz ${ }^{2,3,4, \#}$

$5 \quad{ }^{1}$ Shanghai Key Laboratory of Metabolic Remodeling and Health, Institute of Metabolism \& 6 Integrative Biology, Fudan University, Shanghai, 200433, China. ${ }^{2}$ Lewis-Sigler Institute for 7 Integrative Genomics, Princeton University, Princeton, NJ, 08544, USA. ${ }^{3}$ Department of 8 Chemistry, Princeton University, Princeton, NJ, 08544, USA. ${ }^{4}$ Department of Molecular 9 Biology, Princeton University, Princeton, NJ, 08544, USA. ${ }^{5}$ Lotus Separation LLC, Department 10 of Chemistry, Princeton University, Princeton, NJ, 08544, USA. ${ }^{6}$ Department of Chemistry, The 11 University of Tennessee at Knoxville, Knoxville, TN, 37996, USA. ${ }^{7}$ Department of Computer 12 Science, Princeton University, Princeton, NJ, 08544, USA. \# Corresponding author, e-mail: joshr@princeton.edu

Abstract

16 Liquid chromatography-high resolution mass spectrometry (LC-MS)-based metabolomics 17 aims to identify and quantitate all metabolites, but most LC-MS peaks remain unidentified. 18 Here, we present a global network optimization approach, NetID, to annotate untargeted LC19 MS metabolomics data. The approach aims to generate, for all experimentally observed ion 20 peaks, annotations that match the measured masses, retention times, and (when available) MS/MS fragmentation patterns. Peaks are connected based on mass differences reflecting adducting, fragmentation, isotopes, or feasible biochemical transformations. Global optimization generates a single network linking most observed ion peaks, enhances peak assignment accuracy, and produces chemically-informative peak-peak relationships, including for peaks lacking MS/MS spectra. Applying this approach to yeast and mouse data, we identified five novel metabolites (thiamine derivatives and $\mathrm{N}$-glucosyl-taurine). Isotope tracer studies indicate active flux through these metabolites. Thus, NetID applies existing metabolomic knowledge and global optimization to annotate untargeted metabolomics data, revealing novel metabolites. 


\section{Introduction}

31 Metabolomics provides a snapshot of the concentrations of detectable small-molecule in a biological

32 system. In so doing, it reflects the integrated impact of genetics and environment on metabolism.

33 One important role of metabolomics is annotating previously unknown or underappreciated 34 metabolites. For example, metabolomics facilitated identification of 2-hydroxyglutarate as an 35 oncometabolite, eventually leading to the development of inhibitors of 2-hydroxyglutarate synthesis 36 as anticancer agents ${ }^{1,2}$. Metabolomics also contributed to the identification of a diversity of natural 37 products $^{3,4}$ and disease biomarkers 5 .

38 A common experimental strategy in metabolomics is liquid chromatography-high resolution mass 39 spectrometry (LC-MS). LC-MS metabolomics measures thousands of ion peaks, of which hundreds 40 are associated with known metabolites. A much greater number of peaks, however, still remain 41 unannotated. A common approach to peak annotation is to compare exact mass and either retention 42 time or MS/MS (MS2) fragmentation pattern to authenticated standards. To facilitate such 43 comparisons, extensive molecular structural databases (Pubchem ${ }^{6}, \mathrm{HMDB}^{7}, \mathrm{KEGG}^{8}, \mathrm{ChemSpider}^{9}$ ), 44 MS2 spectral databases (METLIN ${ }^{10}$, GNPS $^{11}$, MassBank ${ }^{12-14}$ and NIST ${ }^{15}$ ) and software (e.g. XCMS ${ }^{16,17}$, 45 GNPS $^{11}$, SIRIUS ${ }^{18}$ and MS-DIAL ${ }^{19}$ ) have been developed. Peaks can also arise from mass spectrometry 46 phenomena, such as adducts, fragments or isotopes of metabolites ${ }^{20-24}$. Such peaks seem to account 47 for at least half of non-background LC-MS features ${ }^{25-27}$. Despite this progress, a great number of 48 unknown peaks remain, and figuring out their identities is a primary challenge in the field.

49 Network analysis, capitalizing on peak-peak relationships to increase annotation scope and accuracy, 50 has been broadly used in metabolomics data annotation. Workflows employing the concept of 51 molecular connectivity have been used to build networks (e.g., GNPS ${ }^{28-30}$, MetDNA ${ }^{31}$, CliqueMS ${ }^{32}$ and 52 others ${ }^{33-35}$ ). Ions connected by either biochemistry or mass spectrometry phenomena often share 53 MS2 fragmentation pattern similarity. While distinct metabolites typically separate 54 chromatographically, ions connected through mass spectrometry phenomena co-elute.

55 Discovery of novel metabolites involves generation of candidate molecular formulae beyond those 56 in current databases. This can be achieved by modifying formulae of known metabolites using 57 characteristic biochemically feasible atom transformations that match observed MS1 mass 58 differences ${ }^{35,36}$ (e.g. 2.016 for $2 \mathrm{H}$ ). Such approaches can be combined with clustering metabolites 59 based on similar MS2 fragmentation patterns in a molecular network, as demonstrated in GNPS and 60 other works ${ }^{28-31,37}$. In a cluster of connected peaks, one known metabolite peak can help to annotate 61 its neighbors, facilitating unknown discovery. One state-of-the-art database-independent method to 62 generate novel candidate molecular formulae (SIRIUS 4.0) combines high-resolution $\mathrm{m} / \mathrm{z}$, natural 63 isotope abundances, and MS2 spectral analyses ${ }^{18}$. 
64 Existing methods generally focus on annotation of either individual peaks or a subnetwork of peaks.

65 All peaks can be sequentially assessed, but individual annotations do not make full use of all available 66 information regarding other peaks in the network. Global network optimization methods, not dealing with peak annotation one-by-one, but instead all at once to take full advantage of the entire available information, hold potential. For example, a Gibbs-sampling statistics approach has been used to incorporate biochemical connections, isotope patterns, and adduct relationships for probabilistic annotations of all peaks in a metabolomics experiment ${ }^{38-40}$. ZODIAC combines a similar approach and the SIRIUS algorithm ${ }^{41}$. Such Gibbs-sampling approaches allow network connections to shift the probabilities of candidate peak annotations, leading to substantially better annotations results ${ }^{41}$. While capable of assessing the probability of all individual candidate annotations in light of their neighbors, broadly defined, such approaches do not guarantee selection of the optimal overall network as a whole.

An alternative computational strategy focusing on optimizing the entire network, as opposed to statistical annotation of individual peaks, is integer linear programming. Given a scoring function, this computational optimization strategy determines the single best annotation for all peaks collectively. Global optimization of large networks of many thousands of peaks is feasible due to linear formulation of the problem, which renders the approach computationally efficient.

Integer linear programming optimization has, to our knowledge, not been previously applied in the context of molecular network analysis. To this end, we present an efficient stand-alone algorithm "NetID". The algorithm optimizes a network of mass spectrometry peak connections based on MS1 mass differences corresponding to gain or loss of relevant chemical moieties and MS2 spectral similarity, in a manner that differentiates biochemical connections from those based on mass spectrometry phenomena, and that incorporates literature data on known metabolites and their retention times. We applied this integer linear programming optimization approach to untargeted metabolomics data from both Baker's yeast and mouse liver. The global optimization step enforces a single formula assignment for each experimentally observed ion peak, increasing annotation 90 accuracy as estimated by a target-decoy strategy ${ }^{42}$. Through these efforts, we provide likely formulae

91 for several hundred putative novel metabolites and confirm the identities of five new metabolites.

\section{Results}

\section{NetID algorithm}

94 NetID involves three computational steps: candidate annotation, scoring, and network optimization 95 (Figure 1). The workflow starts with a peak table that contains a list of peak $\mathrm{m} / \mathrm{z}$, RT, intensity, and 96 (when available) associated MS2 spectra, with background peaks removed by comparing to a process 97 blank sample. Each peak defines a node in the network. In the candidate annotation phase, we match 98 every experimentally measured node $\mathrm{m} / \mathrm{z}$ to formulae in the selected metabolomics database (e.g. 
99 HMDB). Peaks matching to database formula within $10 \mathrm{ppm}$ are assigned as seed nodes with

100 candidate seed formulae, from which we extend edges to build the network.

101 Edges connect two nodes via gain or loss of specific chemical moieties (atoms). The atom differences 102 can occur either due to metabolism (biochemical connection) or due to mass spectrometry 103 phenomena (abiotic connections). For example, a difference of $\mathrm{H}_{2}$ suggests an oxidation/reduction 104 relationship and defines a biochemical edge. A difference of $\mathrm{Na}-\mathrm{H}$ suggests sodium adducting and is 105 a type of abiotic edge (adduct edge). Other atom differences define other types of abiotic 106 connections (isotope or fragment edges). Most atom differences are specific to biochemical, adduct, 107 isotope, or fragment edges, but a few occur in multiple categories. For example, $\mathrm{H}_{2} \mathrm{O}$ loss can be 108 either biochemical (enzymatic dehydration) or abiotic (in-source water loss). By integrating literature 109 and in-house data, we assembled a list of 25 biochemical atom differences and 59 abiotic atom 110 differences which together define all connections in the network (Supplementary Table 1, 2). Using 111 these lists, we make candidate edge annotations such that (i) $\Delta \mathrm{m} / \mathrm{z}$ between the connected nodes 112 matches the atom mass difference and (ii) only co-eluting peaks are connected by abiotic edges. 113 Through the edge extension process starting from the seed nodes, candidate formulae are assigned 114 to nodes outside the initial seeds. A few rounds of edge extension suffice to give thorough coverage 115 (see Methods). Due to finite mass measurement precision, a single node (including a seed node) may 116 be assigned multiple contradictory candidate formulae, which are resolved at the following scoring 117 and optimization step.

118 NetID then scores every candidate node and edge annotation. Candidate node annotations are 119 scored based on precision of $\mathrm{m} / \mathrm{z}$ match to the molecular formula and (when the relevant information 120 is available) precision of retention time match to known metabolite retention time and quality of 121 MS2 spectra match to database structure. In addition, there is a bonus for matching to formula in $122 \mathrm{HMDB}$ and a penalty for unlikely formulae (e.g. containing an uncommon elemental ratio or extreme 123 number of ring and double bond equivalents $)^{43}$. Biochemical edges receive a positive score for MS2 124 spectra similarity between the connected nodes. Abiotic edges are scored based on precision of co125 elution with the parent metabolite, connection type (adduct, isotope, etc.), and features specific to 126 the connection type, such as expected natural abundance for isotope peaks (see Methods and 127 Supplementary Note 2). The overall impact is to assign high scores to those candidate annotations 128 that effectively align the experimentally observed ion peaks with prior metabolomics knowledge.

129 With a score assigned for each candidate node and edge annotation, we formulate the global 130 network optimization problem as that of maximizing the network score with linear constraints that 131 each node and edge has a single annotation and that they are consistent (e.g. peaks connected by $\mathrm{H}_{2}$ 132 edge must have formula differing by $2 \mathrm{H}$ ). Such optimization is readily performed by linear programing 133 with a typical runtime of minutes to hours on a personal computer, and results in an optimal and 134 consistent network annotation. 


\section{Global network optimization}

136 As an example of the utility of global network optimization, where all peaks and connections are 137 simultaneously considered to enhance annotation accuracy, we present an example network 138 containing five peaks (Figure 2A). We first match experimental measurements to the database, 139 assigning node $a$ and node $b$ as seed nodes adenosine monophosphate (AMP, $\mathrm{C}_{10} \mathrm{H}_{14} \mathrm{~N}_{5} \mathrm{O}_{7} \mathrm{P}$ ) and 140 adenosine $\left(\mathrm{C}_{10} \mathrm{H}_{13} \mathrm{~N}_{5} \mathrm{O}_{4}\right)$, respectively. We also identify five possible connections between the five 141 nodes. Two alternative networks are generated by extending from seed assignments. In the left 142 network, node $c$ is annotated as adenosine $\mathrm{HCl}$ adduct $\left(\mathrm{C}_{10} \mathrm{ClH}_{14} \mathrm{~N}_{5} \mathrm{O}_{4}\right)$, whereas in the right network, 143 node $c$ is (mis)annotated as a putative novel metabolite $\left(\mathrm{C}_{9} \mathrm{H}_{14} \mathrm{~N}_{5} \mathrm{O}_{5} \mathrm{P}\right)$ resulting from $\mathrm{CO}_{2}$ loss from 144 AMP. Node $d$ is ${ }^{13} \mathrm{C}$ isotope of node $c$ in both networks. Node $e$ is annotated as ${ }^{37} \mathrm{Cl}$ isotope of node $c$ 145 in the left network, and is unannotated in the right network because there is no $\mathrm{Cl}$ atom in the parent 146 molecule.

147 The left network has higher total node and edge annotation scores than the right network, and thus 148 is selected by NetID. This selection makes sense to an experienced mass spectroscopist: the ${ }^{37} \mathrm{Cl}$ 149 isotope signature in node $e$ indicates that node $c$ should contain $\mathrm{Cl}$. The power of NetID is that it 150 automatically captures such logic, and uses the power of global computational optimization to extend 151 such inferences across the network in an automated manner.

152 We applied the NetID algorithm to yeast and liver datasets, in both positive and negative ionization 153 mode (Figure 2B, Supplementary Fig. 1A). Raw LC-MS data from replicate yeast or liver samples were 154 analyzed together by peak-picking software (El-MAVEN ${ }^{44}$ ) to generate a single list of peaks 155 consistently found for that sample type and ionization mode. Yeast data were MS1 only, while liver 156 data included targeted MS2 spectra. Considering the example of negative mode yeast data with a 157 total of 5,588 non-background peaks, in the candidate annotation step, roughly 1,600 potential 158 formulae were assigned to 1,400 peaks, with about 200 peaks receiving multiple formula annotations. 159 These nodes were connected by just over 50,000 potential edges. Edge extension expanded coverage 160 to over 5,000 nodes with an average of twelve potential formulae each, highlighting the importance 161 of scoring and network optimization to assign proper formulae. After scoring node and edge 162 annotations, global network optimization settled on about 4,800 unique node annotations. About $20 \%$ 163 of the annotated peaks were metabolites (formula corresponding to $\mathrm{M} \pm \mathrm{H}$ monoisotopic peak 164 existed in database), $14 \%$ were putative novel metabolites (formula not in database but with 165 biochemical connection to a metabolite), and the rest were mass spectrometry phenomena, such as 166 adducts, fragments, isotopes. Thus, after thorough background ion removal, we assign a few 167 thousand peaks as likely metabolite ions and the majority as mass spectrometry artifacts. Orthogonal 168 approaches such as credentialing via isotope labeling ${ }^{27}$ similarly assign the majority of peaks as mass 169 spectrometry artifacts, but annotate fewer peaks as likely metabolites than NetID. 
170 The roughly 5000 nodes were connected by about 10,000 edges. Two nodes share each edge, with 171 each node connected by an average of four edges. These edges were roughly evenly split between 172 biochemical and abiotic connections (Figure 2B, Supplementary Fig. 1A,B). More than 90\% of 173 annotated nodes fell into a single dominant connected network (Supplementary Fig. 1C), reflecting 174 most peaks being connected to core metabolism. About $15 \%$ of peaks (737/5588), however, 175 remained unannotated (Figure 2B). These unannotated peaks likely reflect deficiencies in our lists of 176 allowed atom differences, including additional forms of mass spectrometry phenomena. For example, 177 manual examination of the unconnected peaks revealed a dozen nickel adducts of known 178 metabolites (Supplementary Table. 3). The annotated peaks included several hundred formulae for 179 putative novel metabolites (Supplementary Fig. 2, Supplementary Data 1).

\section{Performance validation}

181 We evaluated the performance of the NetID algorithm using the negative mode yeast data (MS1 only). 182 We first employed a target-decoy estimation strategy, in which we intentionally introduce formulae 183 with biologically unreasonable elements, and test whether our annotation strategy effectively avoids 184 annotating peaks with these fake formulae ${ }^{45,46}$. Assessments were made using a number of different 185 metabolite databases $\left(\mathrm{HMDB}^{7}, \mathrm{YMDB}^{47}\right.$, PubChemLite.0.2.0 ${ }^{48}$, and a subset of biopathway related 186 entries in PubChemLite.0.2.0). As expected, the smaller databases yielded fewer false identifications. 187 Importantly, across all of the databases, NetID more effectively selected appropriate formulae (lower 188 false discovery rate) compared to methods considering $\mathrm{m} / \mathrm{z}$ only, node scores only, or both node and 189 edge scores but without global optimization (Figure 2C, Supplementary Fig. 3A).

190 As an orthogonal means of testing the algorithm, we manually curated 314 peaks as known 191 annotations (Supplementary Data 1), and assessed the fraction annotated correctly. Across 192 databases, NetID resulted in more accurate annotations of these gold standard peaks, with the 193 number of incorrect annotations roughly an order of magnitude lower for NetID compared to node 194 or combined node and edge scores without global optimization (Figure 2C, Supplementary Fig. 3B).

\section{Thiamine-derived metabolites}

196 Among the putative novel metabolites in the yeast metabolomics dataset, we found three with total 197 ion current $>10^{5}$ that are connected in a subnetwork around thiamine. Their formulae are $198 \mathrm{C}_{12} \mathrm{H}_{16} \mathrm{~N}_{4} \mathrm{O}_{2} \mathrm{~S}$ (thiamine+O), $\mathrm{C}_{14} \mathrm{H}_{20} \mathrm{~N}_{4} \mathrm{O}_{2} \mathrm{~S}$ (thiamine $+\mathrm{C}_{2} \mathrm{H}_{2} \mathrm{O}$ ) and $\mathrm{C}_{14} \mathrm{H}_{18} \mathrm{~N}_{4} \mathrm{O}_{2} \mathrm{~S}$, (thiamine+ $\mathrm{C}_{2} \mathrm{H}_{4} \mathrm{O}$ ) 199 (Figure 3A, Supplementary Fig 4). These formula assignments and connections were initially obtained 200 without MS2 spectra being available, reflecting the ability of NetID to make accurate formula 201 assignments and connections based on MS1 data (combined with other peak attributes like retention 202 time). While not found in $\mathrm{HMDB}$, thiamine $+\mathrm{O}$ is documented in METLIN as a thiamine oxidation 203 product, so we focused on the other two potential thiamine derivatives. 
MS2 spectra of the putative thiamine $+\mathrm{C}_{2} \mathrm{H}_{2} \mathrm{O}$ and thiamine $+\mathrm{C}_{2} \mathrm{H}_{4} \mathrm{O}$ contained characteristic thiamine fragments. Both contained a classical pyrimidine fragment, with thiamine $+\mathrm{C}_{2} \mathrm{H}_{2} \mathrm{O}$ also containing an acetylated pyrimidine fragment, leading to a probable structure (Figure $3 A, B$ ). The structural assignment is further supported by the presence of an unmodified thiazole fragment. In contrast, thiamine $+\mathrm{C}_{2} \mathrm{H}_{4} \mathrm{O}$ lacked a classical unmodified thiazole fragment, instead showing a thiazole $+\mathrm{C}_{2} \mathrm{H}_{4} \mathrm{O}$ fragment (and a fragment with further water loss) (Figure 3A,B).

210 Isotope tracing experiments further confirmed these two peaks contain thiamine. When fed [U-

$211{ }^{13} \mathrm{C}$ ]glucose as sole carbon source, yeast synthesize thiamine de novo, resulting fully labeled thiamine 212 species, with carbon counts matching the NetID formula assignments (Figure 3C). Adding unlabeled 213 thiamine to the $\left[\mathrm{U}-{ }^{13} \mathrm{C}\right]$ glucose culture media, yeast uptake the unlabeled thiamine, resulting in 214 unlabeled thiamine and $\mathrm{M}+2$ labeled thiamine $+\mathrm{C}_{2} \mathrm{H}_{2} \mathrm{O}$ and thiamine $+\mathrm{C}_{2} \mathrm{H}_{4} \mathrm{O}$ species. Although 215 discovered in yeast, these are conserved metabolites, found also in mammalian samples (Figure 3D).

216 Acetylation is one of the 25 biochemical atom transformations allowed in NetID, while the addition 217 of $\mathrm{C}_{2} \mathrm{H}_{4} \mathrm{O}$ is much less common biochemically. Accordingly, we looked into thiamine metabolism to 218 explore how thiamine $+\mathrm{C}_{2} \mathrm{H}_{4} \mathrm{O}$ might be produced. Thiamine pyrophosphate is an important cofactor 219 in pyruvate dehydrogenase (PDH, the entry step of carbohydrate to TCA cycle) (Figure 3E). The de220 pyrophosphorylation product of thiamine pyrophosphate intermediate in PDH reaction yields 221 thiamine $+\mathrm{C}_{2} \mathrm{H}_{4} \mathrm{O}$ (Figure 3F).

222 Based on this biochemical route, we realized that analogous products could be formed by $\alpha$ 223 ketoglutarate dehydrogenase (thiamine $+\mathrm{C}_{4} \mathrm{H}_{6} \mathrm{O}_{3}$ ) and branched-chain keto acid dehydrogenase 224 (thiamine $+\mathrm{C}_{4} \mathrm{H}_{8} \mathrm{O}$ ) (Figure 3F). Peaks at both of these exact masses were also experimentally observed, 225 with isotope labeling results supporting their being thiamine-derived metabolites (Supplementary 226 Fig. 5). Thus, NetID enabled the discovery of four novel thiamine-derived metabolites that were not 227 present in metabolomics databases (Supplementary Table 4).

\section{$228 \quad \mathbf{N}$-glucosyl-taurine}

229 We similarly carried out NetID annotation of a mouse liver dataset. We observed multiple putative 230 novel metabolite peaks linked to taurine, by apparent glucosylation $\left(+\mathrm{C}_{6} \mathrm{H}_{10} \mathrm{O}_{5}\right)$, palmitylation $231\left(+\mathrm{C}_{16} \mathrm{H}_{30} \mathrm{O}\right)$ and transamination $\left(+\mathrm{O}-\mathrm{NH}_{3}\right)$ (Figure 4A, Supplementary Fig. 6). Like the thiamine-related 232 peaks, these were initially correctly annotated without relying on MS2 data. While all were missing 233 in HMDB, the latter two were found in METLIN: N-palmitoyl taurine $\left(\mathrm{C}_{18} \mathrm{H}_{37} \mathrm{NO}_{4} \mathrm{~S}\right)$ and 234 sulfoacetaldehyde $\left(\mathrm{C}_{2} \mathrm{H}_{4} \mathrm{O}_{4} \mathrm{~S}\right)$. Pubchem contains an entry for $\mathrm{N}$-glucosyl-taurine $\left(\mathrm{C}_{8} \mathrm{H}_{17} \mathrm{NO}_{8} \mathrm{~S}\right)$ as a 235 synthetic chemical but no database previously suggested it being a metabolite. To confirm the 236 structure of the putative taurine glucosylation product $\left(\mathrm{C}_{8} \mathrm{H}_{17} \mathrm{NO}_{8} \mathrm{~S}\right)$, we chemically synthesized $\mathrm{N}$ 237 glucosyl-taurine. Synthetic N-glucosyl-taurine matched the retention time and MS2 fragmentation 238 pattern of the observed $\mathrm{C}_{8} \mathrm{H}_{17} \mathrm{NO}_{8} \mathrm{~S}$ peak (Figure $4 \mathrm{~B}, \mathrm{C}$ ). In liver samples of mice infused with [U- 
$239{ }^{13} \mathrm{C}$ ]glucose, $\mathrm{C}_{8} \mathrm{H}_{17} \mathrm{NO}_{8} \mathrm{~S}$ appeared in $\mathrm{M}+6$ form, suggesting active biosynthesis of $\mathrm{N}$-glucosyl-taurine 240 from circulating glucose (Figure 4D). N-glucosyl-taurine was not observed in yeast extract but was 241 detected in multiple mouse tissues. Search for peaks matching the N-glucosyl-taurine MS2 spectra 242 using MASST identified matches in both mouse and human samples ${ }^{49}$. Quantitation using the 243 synthetic standard shows that liver has the highest level of glucosyl-taurine at $170 \mu \mathrm{M}$ (Figure 4E,

244 Supplementary Fig. 7). This ranks among the few dozen most abundant liver metabolites.

\section{Discussion}

246 The advent of LC-MS metabolomics revealed tens of thousands of metabolite peaks not matching 247 known formulae, raising the possibility that the majority of metabolites remained to be discovered. 248 While the biosphere likely contains many novel metabolites, it has been increasingly recognized that 249 most peaks in typical untargeted metabolomics studies do not arise from novel metabolites, but 250 rather mass spectrometry phenomena. The goal of comprehensively annotating untargeted 251 metabolomics peaks with molecular formulae has, however, remained elusive.

252 One promising strategy for peak annotation involves building networks where nodes are LC-MS peaks 253 (with associated molecular formulae) and edges are atom transformations linking the peaks. Here 254 we advance this strategy by combining metabolomics knowledge with computational global 255 optimization. We explicitly differentiate biochemical connections reflecting metabolic activity and 256 abiotic connections arising from mass spectrometry phenomena. By formulating the peak annotation 257 challenge as a linear program, we identify an optimal network in light of all observed peaks. Rather 258 than weeding out peaks from mass spectrometry phenomena like adducts and natural isotopes, this 259 approach takes advantage of the information embedded in them. It further provides traceable peak260 peak relationships, which illuminate the basis for assigned formulae and suggest candidate structures.

261 Applying this approach to untargeted LC-MS data from yeast and liver samples, we assign formulae 262 to roughly three-quarters of all non-background peaks. In each of positive and negative mode, the 263 annotated peaks cover about 1000 known metabolites, with on average three mass peaks for every 264 metabolite or putative metabolite (e.g. $\mathrm{M}+\mathrm{H}$ plus two adduct or isotope peaks). This leaves a couple 265 thousand unannotated peaks from each LC-MS run. Based on the observed ratio between peaks and 266 metabolites, this likely correspond to hundreds (but not thousands) of unidentified metabolites. The 267 number of actual novel metabolites being detected could potentially be more, due to isomers sharing 268 the same formula as known metabolites, or less, due to novel adducts (e.g. nickel adducts, which we 269 discovered via careful examination of the unannotated peaks) or other mass spectrometry 270 phenomena. Importantly, this approach has already generated likely formulae for many hundreds of 271 putative novel metabolites (Supplementary Fig. 2, Supplementary Data 1), including five species for 272 which we assign structures (Figure 3, 4). 
273 A key benefit of molecular network-based annotation is the ability to assimilate steadily new 274 information ${ }^{28,29}$. MS2 data, which can be collected in either targeted or data-dependent mode, 275 provide fragmentation information to facilitate peak annotation. Due to the global network 276 optimization approach, new MS2 spectra facilitate not only annotation of the ions being fragmented, 277 but also other peaks throughout the network. Other data types can be seamlessly added. For example, 278 compound class categorization based on MS2 data ${ }^{50}$ or retention time prediction ${ }^{51,52}$ can be added 279 to score nodes. Isotope labeling similarity upon feeding different "heavy" nutrients could potentially 280 be added to score edges. Global optimization integrates new information comprehensively with prior 281 knowledge to arrive at optimal annotations. Cycles of experimentation and machine learning hold 282 the potential to identify most unknown metabolites over the coming decade, eventually providing a 283 robust blueprint of the metabolome (Figure 5).

\section{Methods}

285 Yeast metabolomics sample preparation and isotope labeling

286 S. cerevisiae strain FY4 was grown for at least 10 generations in minimal essential media containing $2870.4 \%\left[\mathrm{U}-{ }^{12} \mathrm{C}\right]$ or $\left[\mathrm{U}^{-13} \mathrm{C}\right]$ glucose and $10 \mathrm{mM}$ ammonium sulfate with or without $0.4 \mathrm{mg} / \mathrm{L}$ thiamine 288 hydrochloride ${ }^{53}$. Then, in mid-exponential phase, $5 \mathrm{~mL}$ culture broth $\left(\mathrm{OD}_{600}=0.80\right)$ was filtered and metabolites were extracted using $1 \mathrm{~mL}$ extraction buffer (40:40:20:0.5 290 acetonitrile:methanol:water:formic acid), followed by adding $88 \mu \mathrm{L}$ neutralization buffer $(15 \%$ $291 \mathrm{NH}_{4} \mathrm{HCO}_{3}$ ). The extracts were kept at $-20^{\circ} \mathrm{C}$ for at least $15 \mathrm{~min}$ to precipitate protein before 292 centrifuging at $16,000 \mathrm{~g}$ for $10 \mathrm{~min}$. The supernatant was used for LC-MS analysis.

\section{Murine metabolomics sample preparation and intravenous infusion experiment}

294 Animal studies followed protocols approved by the Princeton University Institutional Animal Care 295 and Use Committee. Twelve-month-old female wild-type C57BL/6 mice (The Jackson Laboratory, Bar 296 Harbor, ME) on standard mouse chow diet were sacrificed by cervical dislocation and tissues quickly 297 dissected and snap frozen in liquid nitrogen with precooled Wollenberger clamp. Frozen samples 298 from liquid nitrogen were then transferred to $-80^{\circ} \mathrm{C}$ freezer for storage. To extract metabolites, 299 frozen liver tissue samples were first weighed ( $20 \mathrm{mg}$ each) and transferred to $2 \mathrm{~mL}$ round-bottom 300 Eppendorf Safe-Lock tubes on dry ice. Samples were then ground into powder with a cryomill 301 machine (Retsch, Newtown, PA) for 30 seconds at $25 \mathrm{~Hz}$, and maintained at cold temperature using 302 liquid nitrogen. For every 25 mg tissues, 922 uL extraction buffer (as above) was added to the tube, 303 vortexed for 10 seconds, and allowed to sit on ice for 10 minutes. Then $78 \mu \mathrm{L}$ neutralization buffer 304 was added and the samples vortexed. The samples were allowed to sit on ice for 20 minutes and then 305 centrifuged at $16,000 \mathrm{~g}$ for $25 \mathrm{~min}$ at $4^{\circ} \mathrm{C}$. The supernatants were transferred to another Eppendorf 306 tube and centrifuged at $16,000 \mathrm{~g}$ for another $25 \mathrm{~min}$ at $4^{\circ} \mathrm{C}$. The supernatants were transferred to 
307 glass vials for LC-MS analysis. A procedure blank was generated identically without tissue, which was

308 used later to remove the background ions.

309 Detailed methods for intravenous infusion of mice have been described previously ${ }^{54}$. Briefly, in vivo 310 infusions were performed on 12-14-week-old C57BL/6 mice pre-catheterized in the right jugular vein 311 (Charles River Laboratories). Mice were kept fasted for $6 \mathrm{~h}$ and then infused for $2.5 \mathrm{~h}$ with [U$312{ }^{13} \mathrm{C}$ ]glucose (200 mM, $0.1 \mu \mathrm{L} / \mathrm{min} / \mathrm{g}$ ). The mouse infusion setup (Instech Laboratories) included a 313 tether and swivel system so that the animal had free movement in the cage. Venous samples were 314 taken from tail bleeds. At the end of the infusion, the mouse was euthanized by cervical dislocation 315 and tissues were collected and extracted as above. Serum metabolites were extracted by adding 100 $316 \mu \mathrm{l}$ methanol to $5 \mu \mathrm{L}$ of serum and centrifuging for $20 \mathrm{~min}$. The supernatant was used for LC-MS 317 analysis.

\section{Glucosyl-taurine synthesis}

319 Glucosyl-taurine synthesis was carried out following previous literature reports with slight modifications ${ }^{55}$. In brief, dry methanol was obtained by distillation of HPLC-grade methanol (Fisher; HPLC grade 0.2 micron filtered) over $\mathrm{CaH}_{2}$ (Acros Organics; ca. 93\% extra pure, 0-2 mm grain size). A

322 flame-dried round-bottom flask equipped with a reflux condenser and stir bar was charged with 2.0 323 g taurine (Alfa Aesar; 99\%), 3.1 g D-glucose (Acros Organics; ACS reagent), and $80 \mathrm{~mL}$ of dry methanol. 324 This mixture was sonicated under an inert atmosphere for $30 \mathrm{~min}$ before being returned to the manifold for the reaction. To the fine-suspension of taurine and glucose in dry methanol at room temperature, $4.0 \mathrm{~mL} 5.4 \mathrm{M}$ sodium methoxide in methanol (Acros Organics) was added via glass syringe. At this point, the suspension began to dissolve and after 30 minutes, gave a clear and colorless solution. The solution was stirred vigorously under an inert atmosphere for 72 hours, which resulted in a faint peach-colored solution. This solution was chilled to $0{ }^{\circ} \mathrm{C}$, and $\sim 200 \mathrm{~mL}$ of absolute ethanol (200 proof) was added and precipitation was allowed to occur at this temperature for 30 minutes. Solvent was then removed by filtration over a glass filter (medium porosity), and washed with $100 \mathrm{~mL}$ of absolute ethanol, affording a fine pale-yellow powder ( $2.4 \mathrm{~g}$; crude material).

NMR was carried out to validate the structure of synthesized N-glucosyl-taurine. Selective TOCSY experiments using DIPSI2 spin-lock and with added chemical shift filter ${ }^{56}$ were run on a Bruker Avance 335 III HD NMR spectrometer equipped with a custom-made QCI-F cryoprobe (Bruker, Billerica, MA) at $336800 \mathrm{MHz}$ and at $295 \mathrm{~K}$ controlled temperature. The sample was dissolved in DMSO-d6. The spectra 337 shown on the plots are results of 200 ms SL mixing, 8 scans each. Data processing (MNova v.14, 338 Mestrelab Research S.L., Santiago de Compostela, Spain) included zero filling, $1 \mathrm{~Hz}$ Gaussian 339 apodization, phase- and baseline correction. NMR analysis suggests that the final crude material 340 contains 5.2\% N-glucosyl-taurine mixed with unreacted substrates (Supplementary Fig. 8). 
342 LC separation was achieved using a Vanquish UHPLC system (Thermo Fisher Scientific) with an

343 Xbridge BEH Amide column $(150 \times 2 \mathrm{~mm}, 2.5 \mu \mathrm{m}$ particle size; Waters). Solvent A is 95:5 water: 344 acetonitrile with $20 \mathrm{mM}$ ammonium acetate and $20 \mathrm{mM}$ ammonium hydroxide at $\mathrm{pH}$ 9.4, and solvent

$345 \mathrm{~B}$ is acetonitrile. The gradient is $0 \mathrm{~min}, 90 \% \mathrm{~B} ; 2 \mathrm{~min}, 90 \% \mathrm{~B} ; 3 \mathrm{~min}, 75 \% ; 7 \mathrm{~min}, 75 \% \mathrm{~B} ; 8 \mathrm{~min}, 70 \%, 9$ $346 \min , 70 \%$ B; $10 \mathrm{~min}, 50 \%$ B; $12 \mathrm{~min}, 50 \%$ B; $13 \mathrm{~min}, 25 \%$ B; $14 \mathrm{~min}, 25 \%$ B; $16 \mathrm{~min}, 0 \% \mathrm{~B}, 20.5 \mathrm{~min}, 0 \%$ 347 B; $21 \mathrm{~min}, 90 \% \mathrm{~B} ; 25 \mathrm{~min}, 90 \% \mathrm{~B}$. Total running time is $25 \mathrm{~min}$ at a flow rate of $150 \mu \mathrm{l} / \mathrm{min}$. LC-MS 348 data were collected on a Q-Exactive Plus mass spectrometer (Thermo Fisher) operating in full scan 349 mode with a MS1 scan range of $\mathrm{m} / \mathrm{z} 70-1000$, and resolving power of 160,000 at $\mathrm{m} / \mathrm{z} 200$. Other MS 350 parameters are as follows: sheath gas flow rate, 28 (arbitrary units); aux gas flow rate, 10 (arbitrary 351 units); sweep gas flow rate, 1 (arbitrary units); spray voltage, $3.3 \mathrm{kV}$; capillary temperature, $320^{\circ} \mathrm{C}$; S352 lens RF level, 65; AGC target, $3 \mathrm{E} 6$ and maximum injection time, $500 \mathrm{~ms}$.

353 MS2 spectra were collected in targeted mode using the PRM function at $25 \mathrm{eV} \mathrm{HCD}$ energy with other 354 instrument settings being resolution 17500, AGC target $10^{6}$, Maximum IT 250 ms, isolation window $3551.5 \mathrm{~m} / \mathrm{z}$. Targeted MS2 data for the thiamine related metabolites were collected for structural 356 identification using similar parameters as above except the HCD energy were set at 20, 35, and $50 \mathrm{eV}$ 357 in a step-CE mode.

\section{Data prepossessing}

359 LC-MS raw data files (.raw) were converted to mzXML format using ProteoWizard ${ }^{57}$. El-MAVEN 360 (version 7.0) was used to generate a peak table containing $\mathrm{m} / \mathrm{z}$, retention time, and intensity for 361 peaks. Parameters for peak picking were the defaults except for the following: mass domain 362 resolution is 10 ppm; time domain resolution is 15 scans; minimum intensity is 1000; minimum peak 363 width is 5 scans. The resulting peak table was exported to a .csv file. Redundant peak entries due to 364 imperfect peak picking process are removed if two peaks are within $0.1 \mathrm{~min}$ and their $\mathrm{m} / \mathrm{z}$ difference 365 are within $2 \mathrm{ppm}$. Background peaks are removed if its intensity in procedure blank sample is $>0.5$ 366 fold of that in biological sample.

367 Targeted MS2 data were extracted from the mzXML files using lab-developed Matlab code 368 (Supplementary Note 1). MS2 spectra may contain interfering product ions from co-eluting isobaric 369 parent ions. These interfering product ions were removed by examining the extracted ion 370 chromatogram (EIC) similarity between the product ions in MS2 data and the parent ion in MS1 data. 371 A Pearson correlation coefficient of 0.8 was used as a cutoff to retain those product ions that has 372 similar EIC as the parent ion. The cleaned MS2 data were exported to Excel files for data input. 373 Although the provided workflow uses targeted MS2 data as input, NetID as currently configured can 374 also handle data-dependent MS2 data, but additional parsing software (under development) is need 375 to convert the large primary data-dependent MS2 files into the NetID input format. 
377 NetID algorithm requires three types of input files: a peak table (in .csv format) recording $\mathrm{m} / \mathrm{z}$, 378 retention time, and intensity for peaks; an atom difference rule table (in .csv format), which in our 379 case consisted of a list of 25 biochemical atom differences and 59 abiotic atom differences which 380 together define all connections in the network (Supplementary Table 1, 2), and metabolite 381 information files containing structures, formulae, $\mathrm{m} / \mathrm{z}$ and MS2 spectra of metabolites from the 382 selected metabolomics database (e.g. HMDB) including retention times of known metabolites under 383 the relevant LC conditions. MS2 data input is optional. For the analysis of yeast and mouse liver 384 datasets in Figure 2-4, structures, formulae, $\mathrm{m} / \mathrm{z}$ and MS2 spectra of metabolites were obtained from 385 the Human Metabolome Database (HMDB, version 4.0) and retention times of selected metabolites 386 were determined through running authentic standards using the above-mentioned LC-MS method 387 (Supplementary data 2). For yeast, no MS2 were used in NetID analysis (MS2 were used post hoc to 388 confirm certain annotations). For liver, targeted MS2 spectra were used (1479 positive and 803 389 negative ionization mode spectra experimentally obtained for previously identified peaks of $>10^{5}$ 390 intensity ${ }^{58}$.

\section{Candidate node and edge annotations}

392 The first module of NetID algorithm is to make candidate annotations for seed nodes, assign 393 candidate annotations for other nodes, and assign candidate edges in the network. Each peak is a 394 node in the network. We compare the experimentally measured $\mathrm{m} / \mathrm{z}$ for each node to those of all metabolite formulae in the selected metabolomics database (e.g. HMDB). When the $\mathrm{m} / \mathrm{z}$ difference 396 is within a predefined tolerance (e.g. 10 ppm), candidate formulae and IDs are assigned to the node, 397 and this node is defined as a primary seed node. Note that assignments to seeds are candidate 398 annotations. A primary seed node can contain multiple candidate formulae and IDs if all are within 399 the $\mathrm{m} / \mathrm{z}$ difference range.

400 Edges connect two nodes via gain or loss of specific atoms. We provided a list of 25 biochemical atom 401 differences and 59 abiotic atom differences which together define all connections in the network 402 (Supplementary Table 1, 2, Supplementary Data 2). Let each of these differences be denoted by $D_{i}$. 403 For each node $u$, if there is a node $v$ such that the difference in the measured $\mathrm{m} / \mathrm{z}$ of the nodes 404 matches one of the those in the list of atom mass differences within a predefined $\mathrm{m} / z_{\text {tol }}$ (e.g. 10 $405 \mathrm{ppm}$ ), we add an edge between $u$ and $v$. That is, if $u_{\mathrm{m} / \mathrm{z}}$ and $v_{\mathrm{m} / \mathrm{z}}$ are the experimentally measured $\mathrm{m} / \mathrm{z}$ 406 for the peaks corresponding to nodes $u$ and $v$ respectively (assuming $v_{\mathrm{m} / \mathrm{z}}>u_{\mathrm{m} / \mathrm{z}}$ for simplicity), then 407 there is an edge between these nodes if there is some difference $D_{i}$ such that

$$
\left|\left(v_{\mathrm{m} / \mathrm{z}}-u_{\mathrm{m} / \mathrm{z}}\right)-D_{i}\right|<v_{\mathrm{m} / \mathrm{z}} \times m / z_{t o l}
$$


409 If $D_{i}$ is an abiotic difference, in order to add an edge, it is additionally required that the retention time 410 between two nodes should be within a predefined $R T_{\text {tol }}$ (e.g. $0.2 \mathrm{~min}$ ). That is, if $u_{\mathrm{RT}}$ and $v_{\mathrm{RT}}$ are the 411 retention times for $u$ and $v$ respectively, then it is required that

$$
\left|v_{\mathrm{RT}}-u_{\mathrm{RT}}\right|<R T_{\text {tol }}
$$

413 For each node, its candidate formulae set will expand due to extending formulae from its neighboring 414 nodes through the edge atom differences. For example, when applying the atom difference of edge $415(u, v)$ on the formula assigned to primary seed node $u$, we can derive a new candidate formula for 416 the connected node $v$. If the derived formula's calculated $\mathrm{m} / \mathrm{z}$ is within a predefined $\mathrm{m} / \mathrm{z}$ tolerance 417 (e.g. $5 \mathrm{ppm}$ ) of node $v^{\prime}$ s measured $\mathrm{m} / \mathrm{z}$, then a new candidate formula is added for node $v$. Iterating 418 the process to all candidate formulae of node $u$ through edge $(u, v)$ will further expand candidate 419 formulae for node $v$.

420 We apply the above extension process to formulae of all primary seed nodes through atom difference 421 edges, and these new candidate formulae themselves can be used for another round of extension. 422 Note that a primary seed node will be treated as the rest of nodes during the subsequent rounds of 423 extension, and may as well be assigned with new formulae. To avoid duplicated efforts in the 424 extension process, we allow formulae of primary seed nodes and biotransformed formulae thereof 425 to be extended through both biotransformation and abiotic atom difference edges, and do not allow 426 abiotic candidate formulae be further extended through biotransformation atom difference edges. 427 The default extension process includes two rounds of biotransformation edge extensions and three 428 rounds of abiotic edge extensions.

429 Each candidate node annotation is defined as (i) metabolite, (ii) putative novel metabolite, or (iii) 430 artifact (nodes can also be unannotated). Specifically, if the elemental formula corresponding to the 431 (de)protonated ion of a monoisotopic peak is found in the employed metabolomics database, this 432 node is defined as a metabolite. If the formula is not found at the employed database, but the node 433 is connected to a metabolite via biochemical connection(s), the node is defined as a putative novel 434 metabolite. Finally, if the node connected only via abiotic connections such as adduct, fragment, or 435 isotope connection(s), it is defined as an artifact. As currently configured, NetID defines metabolite 436 peaks exclusively as (de)protonated ions. In the case that a (de)protonated ion peak is not detected, 437 but related adducts are (e.g. $\left.[\mathrm{M}+\mathrm{Na}]^{+}\right)$, the adducts will remain unannotated (or be misannotated), 438 as there is no procedure for annotating adducts lacking (de)protonated ion peaks.

\section{Scoring in NetID}

440 The second module of NetID algorithm is to score every candidate node and edge annotation 441 assigned in the candidate annotation step. 
442 The node scoring system aims to assign high scores to annotations that align observed ion peaks with 443 known metabolites based on $\mathrm{m} / \mathrm{z}$, retention time, MS2, and/or isotope abundances. Let the set of 444 candidate annotation for node $u$ be denoted as $\left\{a_{1} \ldots a_{i} \ldots a_{m}\right\}$. For each node $u$ and each of its 445 candidate annotation $a_{i}$, let $\mathrm{S}\left(u, a_{i}\right)$ denotes the score of candidate annotation $a_{i}$ for node $u$. $446 \mathrm{~S}\left(u, a_{i}\right)$ is the sum of seven different scoring components, including (a) $\mathrm{S}_{\mathrm{m} / \mathrm{z}}$, a negative score 447 evaluating the difference of between measured $\mathrm{m} / \mathrm{z}$ and the calculated $\mathrm{m} / \mathrm{z}$ of assigned molecular 448 formula; (b) $\mathrm{S}_{\mathrm{RT}}$, a positive score if the measured RT for the peak corresponding to node $u$ matches 449 to a known standard; (c) $\mathrm{S}_{\mathrm{MS} 2}$, a positive score if the measured MS2 spectrum of node $u$ matches 450 the database MS2 spectrum of annotation $a_{i} ;$ (d) $S_{\text {database, a positive score if the annotated formula }}$ $451 a_{i}$ exists in the employed metabolomics database; (e) $S_{\text {missing_isotope, a negative score if an }}$

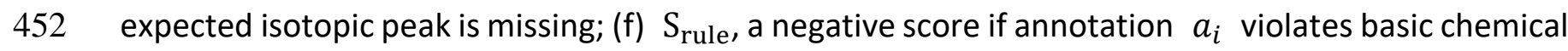

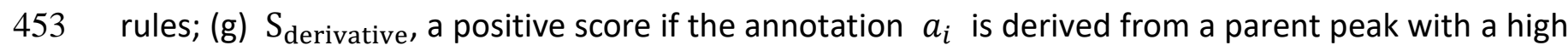
454 score annotation. For details, see Supplementary Note 2.

455 The edge scoring system aims to assign high scores to edge annotations that correctly capture 456 biochemical connections between metabolites (based on MS2 spectra similarity) and abiotic 457 connections between metabolites and their mass spectrometry phenomena derivatives. Biochemical, 458 isotope, adduct, and neural loss edge annotations are the most common types. We also score other 459 less common abiotic connection types appeared in orbitrap data, including oligomers, multi-charge 460 species, heterodimers, in-source fragments of known or unknown metabolites ${ }^{59}$, and ringing artifact 461 peaks surrounding high intensity ions ${ }^{26,60}$.

462 Suppose we consider two nodes $u$ and $v$ that are connected by an edge $(u, v)$. For each pair of nodes $463 u$ and $v$ such that there is an edge $(u, v)$, let the set of candidate formula for node $u$ and $v$ be denoted 464 as $\left\{a_{1} \ldots a_{i} \ldots a_{m}\right\}$ and $\left\{b_{1} \ldots b_{j} \ldots b_{n}\right\}$, respectively, and let the set of candidate atom differences for 465 edge $(u, v)$ be $\left\{D_{1} \ldots D_{k} \ldots D_{l}\right\}$. Let $\mathrm{S}\left(u, v, a_{i}, b_{j}, D_{k}\right)$ be the score of choosing candidate formula $a_{i}$ 466 for node $u$, candidate formula $b_{j}$ for node $v$ and candidate atom difference $D_{k}$ for edge $(u, v)$. Note 467 that $\mathrm{S}\left(u, v, a_{i}, b_{j}, D_{k}\right)$ is set to be 0 if atom difference $D_{k}$ does not represent the formula difference 468 of $a_{i}$ and $b_{j}$.

$$
\mathrm{S}\left(u, v, a_{i}, b_{j}, D_{k}\right)=0, \text { if }\left|a_{i}-b_{j}\right| \neq D_{k}
$$

$\mathrm{S}\left(u, v, a_{i}, b_{j}, D_{k}\right)$ is the sum of four different scoring components, including (a) $\mathrm{S}_{\mathrm{MS} 2 \text { similarity, a }}$ positive score defined for biochemical edges if node $u$ and $v$ have experimental measured MS2

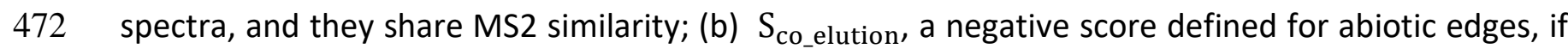
473 the RT of two connected nodes differ more than a threshold (e.g. $0.05 \mathrm{~min}$ ); (c) $\mathrm{S}_{\mathrm{type}}\left(u, v, a_{i}, b_{j}, D_{k}\right)$, 474 a non-negative score defined for all edges, depending on the connection type of edge, which is 475 defined by $D_{k}$, including biotransformation, adduct, isotope, and fragment edges, and optionally 
including oligomer and multi-charge species, heterodimer, in-source fragments and ringing artifacts

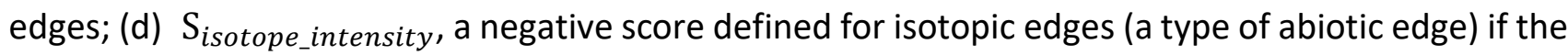

478 measured isotope peaks deviate from expected natural abundance. For details, see Supplementary Note 2.

\section{Global network optimization using linear programing}

481 The third module of NetID algorithm is to perform global network optimization. Using scores assigned 482 for each candidate node and edge annotation, our goal is to find formula annotations for each node 483 and edge so as to maximize the sum of their scores across the network under the constraints that 484 each node is assigned a single annotation, and that the network annotation is consistent. When a 485 node has multiple candidate node annotations shared with same formula (e.g. isomers), the one with 486 highest score (better MS2 match or RT match) is selected. When equal scores happen, the candidate 487 annotation that appears first in the metabolite list from database is reported as a default. We use 488 linear programming to solve this optimization problem, as described next.

489 For each node $u$ and each of its candidate formula $a_{i}$, we define a node binary decision variable $490 x_{u, a_{i}}$ to denote whether candidate formula $a_{i}$ is selected as the annotation for node $u$. That is,

$$
x_{u, a_{i}}=1 \text {, if node } u \text { is annotated with formula } a_{i}
$$

493 For each edge, we define a binary decision variable $c_{u, v, a_{i}, b_{j}, D_{k}}$ to denote whether candidate

494 formulae $a_{i}$ and $b_{j}$ are chosen for nodes $u$ and $v$, and the candidate atom difference $D_{k}$

495 corresponds to the formula difference of candidate formulae $a_{i}$ and $b_{j}$ of the connected nodes $u$ 496 and $v$. That is,

$$
c_{u, v, a_{i}, b_{j}, D_{k}}=1, \text { if }\left|a_{i}-b_{j}\right|=D_{k}
$$

499 We constrain the optimization so that each node has a single annotation, and an edge exists only if 500 the atom difference of that edge annotation matches the formula difference of nodes. For 501 computational purposes, nodes may also receive "blank" or "no formula" annotation (we refer to 502 such nodes in elsewhere in the text as "unannotated"). The node and edge binary variables must 503 satisfy 


$$
c_{u, v, a_{i}, b_{j}, D_{k}} \geq x_{u, a_{i}}+x_{v, b_{j}}-1
$$

507 For all variables defined above, we add the constraints that they are either 1 or 0 , representing the candidate annotation is selected or not selected, respectively, in the network.

509 With scores of each candidate node and edge annotation, the objective for the optimization is to 510 determine all variables $x_{u, a}$ and $c_{u, v, a, b, D}$ so as to maximize the sum of all node scores and edge scores in a network while satisfying the above constraints.

$$
\text { Maximize: } \sum x_{u, a} \times \mathrm{S}(u, a)+\sum c_{u, v, a, b, D} \times \mathrm{S}(u, v, a, b, D)
$$

513 The optimization result provides a string of binary numbers that denote if a candidate node or edge

514 annotation is selected for the global optimal network. IBM ILOG CPLEX Optimization Studio (Version

51512.8 .0 or later) is used to solve the linear programing problem. For the reported datasets and using 516 the default parameter settings, optimization finishes within an hour on a standard laptop 517 (Supplementary table 5). Depending on the number of peaks in data tables, the entries in the atom 518 difference tables, the choice of reference compound databases, and the parameters involved in 519 scoring, runtimes during internal testing ranged from minutes to hours.

\section{Evaluation of NetID}

521 After running the candidate annotation and network extension process as usual for NetID, we 522 compared four different annotation selection methods: (i) $\mathrm{m} / \mathrm{z}$ only, selecting the candidate 523 annotation with closest $\mathrm{m} / \mathrm{z}$ to the measured $\mathrm{m} / \mathrm{z}$; (ii) node scores, selecting the candidate 524 annotation with highest candidate node score (as per usual NetID scoring rules); (iii) node + edge scores, adding half of a candidate edge score to each node score of the two connected candidate node annotations, and selecting the candidate annotation with highest combined score; (iv) NetID optimization, using the candidate node and edge score, and selecting the candidate annotation

528 from global optimization.

529 We employed a target-decoy strategy to estimate false discovery rate ${ }^{45,46}$. The target library is the 530 compound library we use for annotation, including HMDB (human metabolomics database), PBCM 531 (PubChemLite.0.2.0) ${ }^{48}$, PBCM_BIO (a subset of biopathway related entries in PubChemLite.0.2.0) 532 and YMDB (yeast metabolomics database) ${ }^{47}$. The decoy formula was generated by adding an 533 implausible element adduct to a formula from target library. These implausible elements are those 534 not in any formulae in database, namely, $\mathrm{He}, \mathrm{Be}, \mathrm{Ne}, \mathrm{Sc}, \mathrm{Kr}, \mathrm{Rb}, \mathrm{Sr}, \mathrm{Y}, \mathrm{Zr}, \mathrm{Nb}, \mathrm{Mo}, \mathrm{Ru}, \mathrm{Rh}, \mathrm{Pd}, \mathrm{Ag}, \mathrm{Cd}$, 535 In, Sn, Sb, Te, Xe, Cs, Ba, La, Ce, Pr, Nd, Sm, Eu, Gd, Tb, Ho, Er, Tm, Yb, Lu, Hf, Ta, W, Re, Os, Ir, Pt, $536 \mathrm{Au}, \mathrm{Hg}, \mathrm{Tl}, \mathrm{Pb}, \mathrm{Bi}$, Th and $\mathrm{U}$. Applying the decoy formula generation process (i.e. adding a single 
537 randomly selected implausible element in place of hydrogen) to all unique formulae in a target 538 compound library gives the corresponding decoy library. The combined target library and decoy

539 library were used for annotation. Any annotation containing an implausible element is considered a 540 false positive (FP) result. The number of FP results from target library is estimated to be similar to 541 that from decoy library, because 1:1 ratio of target formulae and decoy formulae were used. That is

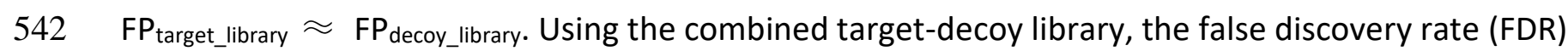
543 is estimated to be $F P_{\text {decoy_library }}$ / ( $T$ - FP decoy_library), where $\mathrm{T}$ is the total number of annotations. The 544 decoy library generation process was repeated ten times for each database.

545 We manually annotated 314 peaks in the yeast negative mode dataset (Supplementary data 1). 546 Using these annotations as a ground truth, we evaluated the fraction of correct annotation for the 547 four different annotation methods above. Peak annotations matching to the ground truth formulae, 548 including adduct, isotope formulae, are counted as correct, and peaks that are not annotated or 549 their annotations did not match are counted as incorrect. The annotation process used 1:1 target550 decoy library and was repeated 10 times as above.

\section{$551 \quad$ Visualization}

552 We provide an interactive Shiny R app to visualize and explore the NetID output network. In 553 addition, NetID outputs two .csv files (cyto_node.csv and cyto_edges.csv) that are compatible with 554 the general network visualization software Cytoscape. The interactive Shiny R app and detailed user 555 guide are available at GitHub (https://github.com/LiChenPU/NetID).

\section{Data availability}

557 All raw data, including the yeast, the mouse studies, the ${ }^{13} \mathrm{C}$ studies and over 2000 targeted MS2 files 558 collected from the liver data in mzXML formats were deposited in MassIVE (ID = MSV000087434). 559 MS2 spectra of reported novel metabolites are included in Supplementary Data 3. R code for 560 generating NetID statistics and performing FDR analysis in Figure 2 and Supplementary Fig 1 are 561 provided in GitHub (https://github.com/LiChenPU/NetID). Peak table for yeast data negative mode, 562 including NetID annotation results, putative novel metabolite list, and manual curation results are 563 provided in Supplementary Data 1. Exemplary peak table from the yeast dataset, atom difference 564 rule table, HMDB compound list (customized to contain relevant information), and an in-house 565 retention time list for known metabolites are provided in Supplementary Data 2.

\section{Code availability}

567 NetID was developed mainly in R, and used a mixture of IBM ILOG CPLEX Optimization Studio, 568 Matlab and Python. NetID code and example files are available for non-commercial use in GitHub at https://github.com/LiChenPU/NetID, under the GNU General Public License v3.0. User guide, parameter explanation and pseudocode are provided in Supplementary Notes 1, 2, and 3. 


\section{Acknowledgement}

572 This work was supported by a Department of Energy (DOE) grant (no. DE-SC0012461 to J.D.R.), the 573 Center for Advanced Bioenergy and Bioproducts Innovation (grant no. DE-SC0018420, subcontract to 574 J.D.R.) and NIH grant R50CA211437 to W.L. M.R.M is funded by the Howard Hughes Medical Institute 575 and Burroughs Wellcome Fund via the PDEP and Hanna H. Gray Fellows Programs. We thank Istvan 576 Pelczer at NMR facility of Department of Chemistry at Princeton University for the NMR analysis, the 577 Metabolomics \& Lipidomics Mass Spectrometry Core Facility of IMIB at Fudan University for 578 additional mass spectrometry support, and X. Su for scientific discussion and help. The Center for 579 Advanced Bioenergy and Bioproducts Innovation and the Center for Bioenergy Innovation are both 580 U.S. Department of Energy Bioenergy Research Centers supported by the Office of Biological and 581 Environmental Research in the DOE Office of Science. Any opinions, findings, and conclusions or 582 recommendations expressed in this publication are those of the authors and do not necessarily 583 reflect the views of the U.S. Department of Energy.

\section{Competing interests}

585 The authors declare no competing interests.

\section{Author contributions}

587 L.C., M.S. and J.D.R. conceived the project. L.C., X.X. and Z.C. wrote the NetID algorithm code. W.L., 588 L.W., X.Z., A.C. M.M. performed experiments on mouse. L.W., W.L. and L.C. performed experiments 589 on yeast. L.C., W.L., L.W. and X.X. analyzed LC-MS and LC-MS/MS data. X.T., A.M. and Y.S. contributed 590 to coding development. B.K., A.M.L., and S.R.C. synthesized taurine-related compounds. L.C. and 591 J.D.R. wrote the manuscript. All authors discussed the results and commented on the manuscript. 
595 Figure 1. A global network optimization approach for untargeted metabolomics data annotation

596 (NetID). The input data are LC-MS peaks with $\mathrm{m} / \mathrm{z}$, retention times, intensities and optional MS2 597 spectra. The output is a molecular network with peaks (nodes) assigned with unique formulae and 598 connected by edges reflecting atom differences arising either through metabolism (biochemical 599 connection) or mass spectrometry phenomenon (abiotic connection). Peaks are classified as 600 "metabolite" ( $\mathrm{M}+\mathrm{H}$ or $\mathrm{M}-\mathrm{H}$ peak of formula found in selected metabolomics database, e.g. HMDB), 601 "putative novel metabolite" (formula not found in database but with biochemical connection to a 602 metabolite), or "artifact" (only abiotic connection to a metabolite). NetID algorithm involves three 603 steps. Candidate annotation first matches peaks to database formulae. These seed annotations are 604 then extended through edges to cover most nodes, with the majority of nodes receiving multiple 605 formula annotations. Each node and edge annotation are then scored based on match to known 606 masses, retention times, and MS/MS fragmentation patterns. Global network optimization 607 maximizes sum of node scores and edge scores, while enforcing a unique formula for each node and 608 unique transformation relationship for each edge.

609 Figure 2. Utility of global network optimization. (A) An example network demonstrating the value 610 of the global optimization step in NetID. Node $a$ and node $b$ match database formulae and are 611 connected by an edge of phosphate $\left(\mathrm{HPO}_{3}\right)$. Node $c$ can be connected to either node $a$ or node $b$ 612 through mutually incompatible annotations, resulting in two different candidate networks. The table 613 below the two candidate networks shows the annotations and scoring criteria for each, with the left 614 network preferred for more good node and edge annotations. (B) Summary table of NetID 615 annotations of negative and positive mode LC-MS data from Baker's yeast and mouse liver. (C) False 616 discovery rate estimated using target-decoy strategy. Each individual data point (circle) is from a 617 different randomized decoy library ( $n=10$ randomized libraries were tested). (D) Fraction of 314 618 manually curated "ground truth" annotations made correctly (using the 10 libraries). Boxes show 619 median and interquartile range.

620 Figure 3. NetID reveals thiamine-derived metabolites in yeast. (A) Subnetwork surrounding 621 thiamine. Nodes, connections, and formulae are direct output of NetID. Boxes with structures were 622 manually added. (B) MS2 spectra of thiamine, thiamine $+\mathrm{C}_{2} \mathrm{H}_{2} \mathrm{O}$, and thiamine $+\mathrm{C}_{2} \mathrm{H}_{4} \mathrm{O}$, with proposed 623 structures of the major fragments. (C) Labeling fraction of thiamine and its derivatives, in [U$624{ }^{13} \mathrm{C}$ ]glucose with and without unlabeled thiamine in the medium $(n=5)$. (D) The thiamine derivatives 625 are also found in mouse tissues and urine $(n=3)$. (E) Proposed mechanism for formation of 626 thiamine $+\mathrm{C}_{2} \mathrm{H}_{4} \mathrm{O}$. Pyruvate dehydrogenase (PDH) decarboxylates pyruvate, and adds the resulting $627\left[\mathrm{C}_{2} \mathrm{H}_{4} \mathrm{O}\right]$ unit (in red) to thiamine. (F) The same enzymatic mechanism occurs in oxoglutarate 628 dehydrogenase (OGDH) and branched-chain $\alpha$-ketoacid dehydrogenase complex (BCKDC), and 
generates thiamine $+\mathrm{C}_{4} \mathrm{H}_{6} \mathrm{O}_{3}$ and thiamine $+\mathrm{C}_{4} \mathrm{H}_{8} \mathrm{O}$ respectively. Bar represents mean values and error bar indicates s.d. in (C) and s.e. in (D).

Figure 4. NetID discovers mammalian taurine derivatives. (A) Subnetwork surrounding taurine from mouse liver extract data. Nodes, connections, and formulae are direct output of NetID. Boxes with structures were manually added. (B) LC-MS chromatogram of $\mathrm{N}$-glucosyl-taurine standard and the putative glucosyl-taurine from liver extract. (C) Top 10 abundant ion peaks in MS2 spectrum of glucosyl-taurine peak from liver extract (top), and synthetic N-glucosyl-taurine standard (bottom). (D) Isotope labeling pattern of putative glucosyl-taurine in mice, infused via jugular vein catheter for $2 \mathrm{~h}$ with $\left[\mathrm{U}-{ }^{13} \mathrm{C}\right]$ glucose $(\mathrm{n}=3)$. (E) Absolute $\mathrm{N}$-glucosyl-taurine concentration in murine serum and tissues $(n=3)$. Bar represents mean values and error bar indicates s.d. in (D) and s.e. in (E).

Figure 5. NetID applies global optimization for metabolomics data annotation and metabolite discovery.

\section{Reference}

1. DiNardo, C. D. et al. Durable Remissions with Ivosidenib in IDH1-Mutated Relapsed or Refractory AML. N. Engl. J. Med. 378, 2386-2398 (2018).

2. Dang, L. et al. Cancer-associated IDH1 mutations produce 2-hydroxyglutarate. Nature 462, 739 (2009).

3. Doroghazi, J. R. et al. A roadmap for natural product discovery based on large-scale genomics and metabolomics. Nature Chemical Biology 10, 963-968 (2014).

4. Aron, A. T. et al. Reproducible molecular networking of untargeted mass spectrometry data using GNPS. Nature Protocols 15, 1954-1991 (2020).

5. Johnson, C. H., Ivanisevic, J. \& Siuzdak, G. Metabolomics: beyond biomarkers and towards mechanisms. Nature Reviews Molecular Cell Biology 17, 451-459 (2016).

6. Kim, S. et al. PubChem 2019 update: improved access to chemical data. Nucleic Acids Res 47, D1102-D1109 (2019).

7. Wishart, D. S. et al. HMDB 4.0: the human metabolome database for 2018. Nucleic Acids Res 46, D608-D617 (2018).

8. Kanehisa, M., Sato, Y., Kawashima, M., Furumichi, M. \& Tanabe, M. KEGG as a reference resource for gene and protein annotation. Nucleic Acids Res 44, D457-D462 (2016).

9. Pence, H. E. \& Williams, A. ChemSpider: An Online Chemical Information Resource. J. Chem. Educ. 87, 1123-1124 (2010).

10. Xue, J., Guijas, C., Benton, H. P., Warth, B. \& Siuzdak, G. METLIN MS2 molecular standards database: a broad chemical and biological resource. Nat Methods 17, 953-954 (2020). 
11. Wang, M. et al. Sharing and community curation of mass spectrometry data with Global Natural Products Social Molecular Networking. Nature Biotechnology 34, 828-837 (2016).

12. Tsugawa, H. et al. Hydrogen Rearrangement Rules: Computational MS/MS Fragmentation and Structure Elucidation Using MS-FINDER Software. Anal. Chem. 88, 7946-7958 (2016).

13. Horai, H. et al. MassBank: a public repository for sharing mass spectral data for life sciences. $J$. Mass Spectrom. 45, 703-714 (2010).

14. MassBank | MassBank Europe Mass Spectral DataBase. https://massbank.eu/MassBank/.

15. sherena.johnson@nist.gov. NIST Standard Reference Database 1A. NIST https://www.nist.gov/srd/nist-standard-reference-database-1a (2014).

16. Tautenhahn, R., Patti, G. J., Rinehart, D. \& Siuzdak, G. XCMS Online: A Web-Based Platform to Process Untargeted Metabolomic Data. Anal. Chem. 84, 5035-5039 (2012).

17. Forsberg, E. M. et al. Data processing, multi-omic pathway mapping, and metabolite activity analysis using XCMS Online. Nature Protocols 13, 633-651 (2018).

18. Dührkop, K. et al. SIRIUS 4: a rapid tool for turning tandem mass spectra into metabolite structure information. Nature Methods 16, 299-302 (2019).

19. Tsugawa, H. et al. A cheminformatics approach to characterize metabolomes in stable-isotopelabeled organisms. Nature Methods 16, 295 (2019).

20. Stricker, T., Bonner, R., Lisacek, F. \& Hopfgartner, G. Adduct annotation in liquid chromatography/high-resolution mass spectrometry to enhance compound identification. Anal Bioanal Chem 413, 503-517 (2021).

21. Kuhl, C., Tautenhahn, R., Böttcher, C., Larson, T. R. \& Neumann, S. CAMERA: An Integrated Strategy for Compound Spectra Extraction and Annotation of Liquid Chromatography/Mass Spectrometry Data Sets. Anal. Chem. 84, 283-289 (2012).

22. Domingo-Almenara, X. et al. Autonomous METLIN-Guided In-source Fragment Annotation for Untargeted Metabolomics. Anal. Chem. 91, 3246-3253 (2019).

23. Broeckling, C. D., Afsar, F. A., Neumann, S., Ben-Hur, A. \& Prenni, J. E. RAMClust: A Novel Feature Clustering Method Enables Spectral-Matching-Based Annotation for Metabolomics Data. Anal. Chem. 86, 6812-6817 (2014).

24. Domingo-Almenara, X., Montenegro-Burke, J. R., Benton, H. P. \& Siuzdak, G. Annotation: A Computational Solution for Streamlining Metabolomics Analysis. Anal. Chem. 90, 480-489 (2018).

25. Sindelar, M. \& Patti, G. J. Chemical Discovery in the Era of Metabolomics. J. Am. Chem. Soc. 142, 9097-9105 (2020).

26. Wang, L. et al. Peak Annotation and Verification Engine for Untargeted LC-MS Metabolomics. Anal. Chem. 91, 1838-1846 (2019). 
27. Mahieu, N. G., Huang, X., Chen, Y.-J. \& Patti, G. J. Credentialing Features: A Platform to Benchmark and Optimize Untargeted Metabolomic Methods. Anal. Chem. 86, 9583-9589 (2014).

28. Schmid, R. et al. Ion Identity Molecular Networking in the GNPS Environment.

$$
\text { http://biorxiv.org/lookup/doi/10.1101/2020.05.11.088948 (2020) }
$$
doi:10.1101/2020.05.11.088948.

29. Nothias, L.-F. et al. Feature-based molecular networking in the GNPS analysis environment. Nat Methods 17, 905-908 (2020).

30. da Silva, R. R. et al. Propagating annotations of molecular networks using in silico fragmentation. PLOS Computational Biology 14, e1006089 (2018).

31. Shen, X. et al. Metabolic reaction network-based recursive metabolite annotation for untargeted metabolomics. Nature Communications 10, 1516 (2019).

32. Senan, O. et al. CliqueMS: A computational tool for annotating in-source metabolite ions from LC-MS untargeted metabolomics data based on a coelution similarity network. 8.

33. Alden, N. et al. Biologically Consistent Annotation of Metabolomics Data. Anal. Chem. 89, 13097-13104 (2017).

34. Del Carratore, F. et al. Integrated Probabilistic Annotation: A Bayesian-Based Annotation Method for Metabolomic Profiles Integrating Biochemical Connections, Isotope Patterns, and Adduct Relationships. Anal. Chem. (2019) doi:10.1021/acs.analchem.9b02354.

35. Yu, M. \& Petrick, L. Untargeted high-resolution paired mass distance data mining for retrieving general chemical relationships. Commun Chem 3, 157 (2020).

36. Watrous, J. et al. Mass spectral molecular networking of living microbial colonies. PNAS 109, E1743-E1752 (2012).

37. Hooft, J. J. J. van der, Wandy, J., Barrett, M. P., Burgess, K. E. V. \& Rogers, S. Topic modeling for untargeted substructure exploration in metabolomics. PNAS 113, 13738-13743 (2016).

38. Del Carratore, F. et al. Integrated Probabilistic Annotation: A Bayesian-Based Annotation Method for Metabolomic Profiles Integrating Biochemical Connections, Isotope Patterns, and Adduct Relationships. Anal. Chem. 91, 12799-12807 (2019).

39. Rogers, S., Scheltema, R. A., Girolami, M. \& Breitling, R. Probabilistic assignment of formulas to mass peaks in metabolomics experiments. Bioinformatics 25, 512-518 (2009).

40. Daly, R. et al. MetAssign: probabilistic annotation of metabolites from LC-MS data using a Bayesian clustering approach. Bioinformatics 30, 2764-2771 (2014).

41. Ludwig, M. et al. Database-independent molecular formula annotation using Gibbs sampling through ZODIAC. Nature Machine Intelligence 2, 629-641 (2020).

42. Palmer, A. et al. FDR-controlled metabolite annotation for high-resolution imaging mass spectrometry. Nature Methods 14, 57-60 (2017). 
43. Kind, T. \& Fiehn, O. Seven Golden Rules for heuristic filtering of molecular formulas obtained by accurate mass spectrometry. BMC Bioinformatics 8, 105 (2007).

44. Melamud, E., Vastag, L. \& Rabinowitz, J. D. Metabolomic Analysis and Visualization Engine for LC-MS Data. Anal. Chem. 82, 9818-9826 (2010).

45. Palmer, A. et al. FDR-controlled metabolite annotation for high-resolution imaging mass spectrometry. Nat Methods 14, 57-60 (2017).

46. Käll, L., Storey, J. D., MacCoss, M. J. \& Noble, W. S. Assigning Significance to Peptides Identified by Tandem Mass Spectrometry Using Decoy Databases. J. Proteome Res. 7, 29-34 (2008).

47. Jewison, T. et al. YMDB: the Yeast Metabolome Database. Nucleic Acids Research 40, D815D820 (2012).

48. Bolton, E. \& Schymanski, E. PubChemLite tier0 and tier1. (2020) doi:10.5281/zenodo.3611238.

49. Wang, M. et al. Mass spectrometry searches using MASST. Nat Biotechnol 1-4 (2020) doi:10.1038/s41587-019-0375-9.

50. Dührkop, K. et al. Systematic classification of unknown metabolites using high-resolution fragmentation mass spectra. Nat Biotechnol (2020) doi:10.1038/s41587-020-0740-8.

51. Bonini, P., Kind, T., Tsugawa, H., Barupal, D. K. \& Fiehn, O. Retip: Retention Time Prediction for Compound Annotation in Untargeted Metabolomics. Anal. Chem. 92, 7515-7522 (2020).

52. Bach, E., Szedmak, S., Brouard, C., Böcker, S. \& Rousu, J. Liquid-chromatography retention order prediction for metabolite identification. Bioinformatics 34, i875-i883 (2018).

53. Xu, Y.-F. et al. Discovery and Functional Characterization of a Yeast Sugar Alcohol Phosphatase. ACS Chem. Biol. 13, 3011-3020 (2018).

54. Hui, S. et al. Glucose feeds the TCA cycle via circulating lactate. Nature 551, 115-118 (2017).

55. Cho, H. J., You, J. S., Chang, K. J., Kim, K. S. \& Kim, S. H. Anti-adipogenic Effect of TaurineCarbohydrate Derivatives. Bulletin of the Korean Chemical Society 35, 1863-1866 (2014).

56. Robinson, P. T., Pham, T. N. \& Uhrín, D. In phase selective excitation of overlapping multiplets by gradient-enhanced chemical shift selective filters. Journal of Magnetic Resonance 170, 97103 (2004).

57. Chambers, M. C. et al. A Cross-platform Toolkit for Mass Spectrometry and Proteomics. Nat Biotechnol 30, 918-920 (2012).

58. Lu, W. et al. Improved Annotation of Untargeted Metabolomics Data through Buffer Modifications That Shift Adduct Mass and Intensity. Anal. Chem. 92, 11573-11581 (2020).

59. Xue, J. et al. Enhanced in-Source Fragmentation Annotation Enables Novel Data Independent Acquisition and Autonomous METLIN Molecular Identification. Anal. Chem. 92, 6051-6059 (2020). 
bioRxiv preprint doi: https://doi.org/10.1101/2021.01.06.425569; this version posted June 16, 2021. The copyright holder for this preprint (which was not certified by peer review) is the author/funder, who has granted bioRxiv a license to display the preprint in perpetuity. It is made available under aCC-BY-NC-ND 4.0 International license.

769 60. Mitchell, J. M. et al. New methods to identify high peak density artifacts in Fourier transform 770 mass spectra and to mitigate their effects on high-throughput metabolomic data analysis. Metabolomics 14, 125 (2018). 


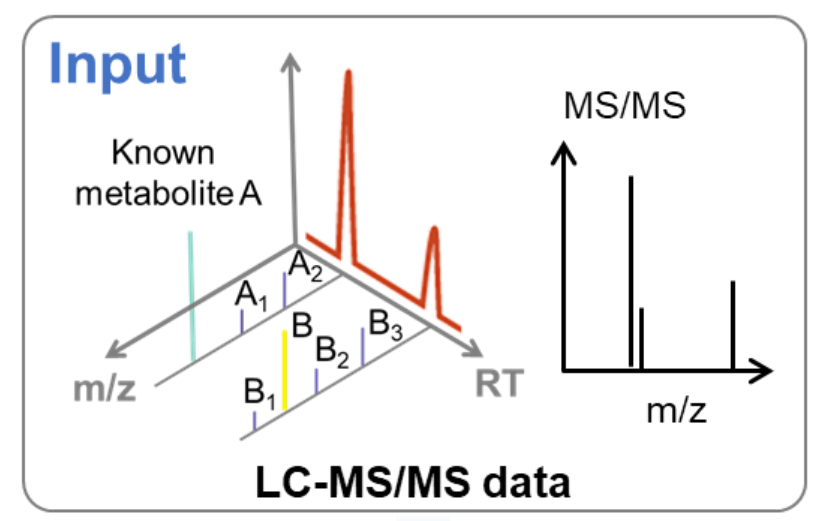

\section{Output}

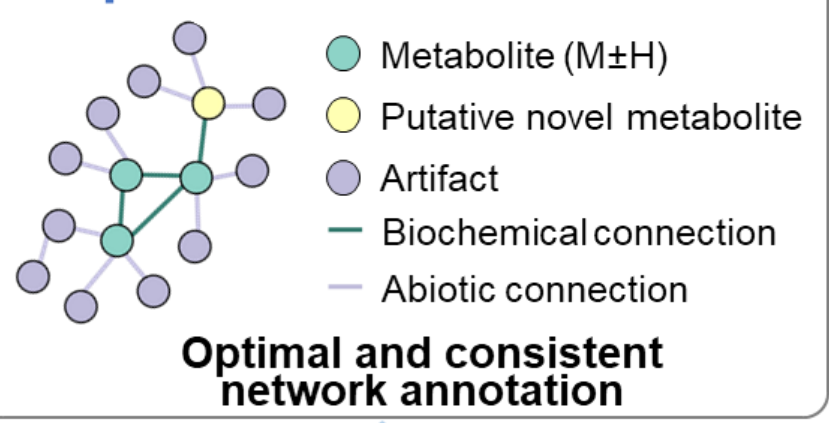

NetID

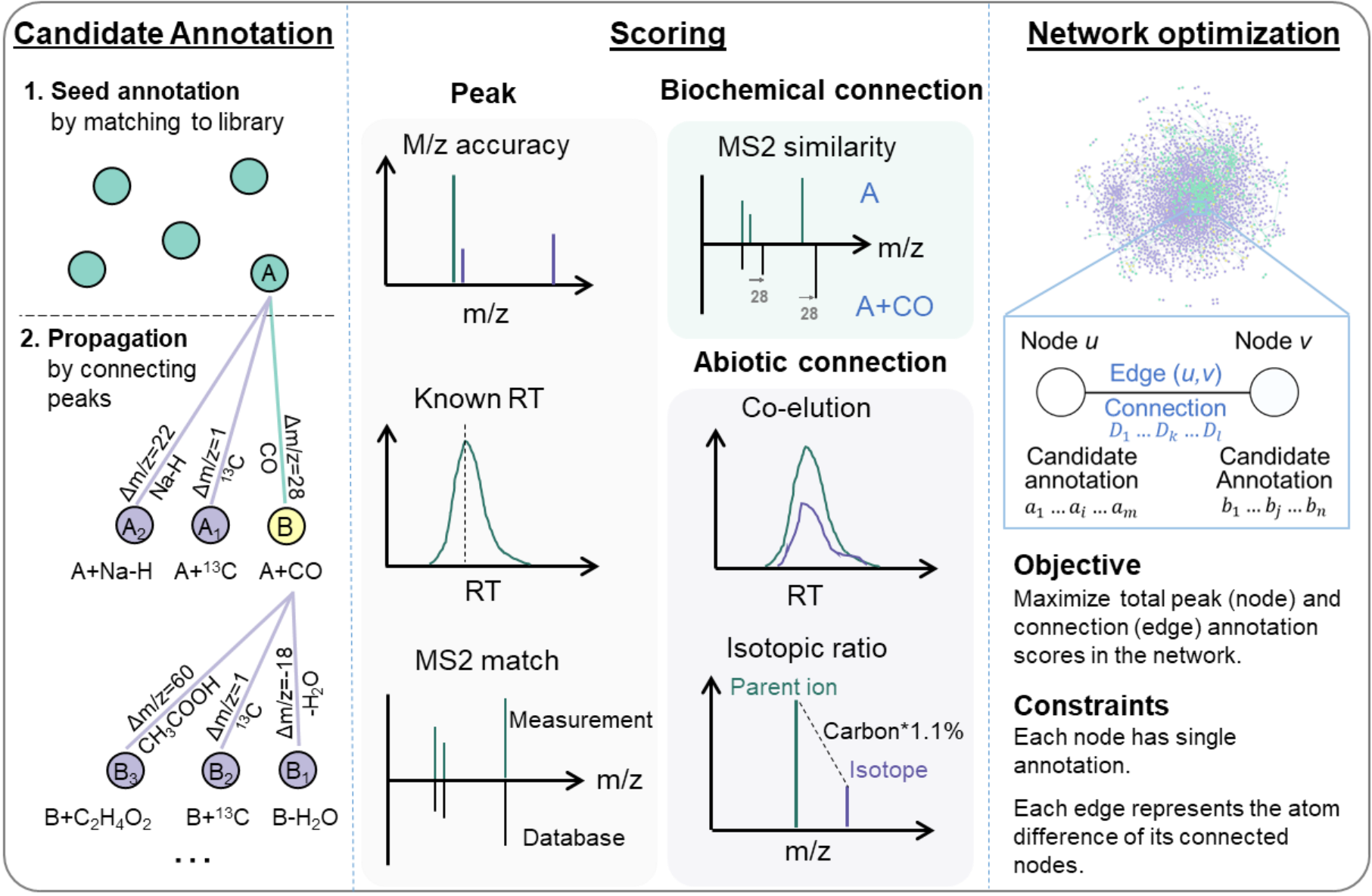

Figure 1. A global network optimization approach for untargeted metabolomics data annotation (NetID). The input data are LC-MS peaks with $\mathrm{m} / \mathrm{z}$, retention times, intensities and optional MS2 spectra. The output is a molecular network with peaks (nodes) assigned with unique formulae and connected by edges reflecting atom differences arising either through metabolism (biochemical connection) or mass spectrometry phenomenon (abiotic connection). Peaks are classified as "metabolite" (M+H or M-H peak of formula found in selected metabolomics database, e.g. HMDB), "putative novel metabolite" (formula not found in database but with biochemical connection to a metabolite), or "artifact" (only abiotic connection to a metabolite). NetID algorithm involves three steps. Candidate annotation first matches peaks to database formulae. These seed annotations are then extended through edges to cover most nodes, with the majority of nodes receiving multiple formula annotations. Each node and edge annotation are then scored based on match to known masses, retention times, and MS/MS fragmentation patterns. Global network optimization maximizes sum of node scores and edge scores, while enforcing a unique formula for each node and unique transformation relationship for each edge. 
A

Metabolite peaks $(\mathrm{M} \pm \mathrm{H})$
Putativenovel
metabolite peaks
Artifact peaks
Unannotated peaks
- Biochemical connection
- Abiotic connection

$\mathrm{C}_{10} \mathrm{H}_{14} \mathrm{~N}_{5} \mathrm{O}_{7} \mathrm{P}$ (a) $-\frac{79.9663}{4_{3} 9_{89_{8}}}-\frac{\mathrm{HPO}_{3}}{35.9767}$ (b)
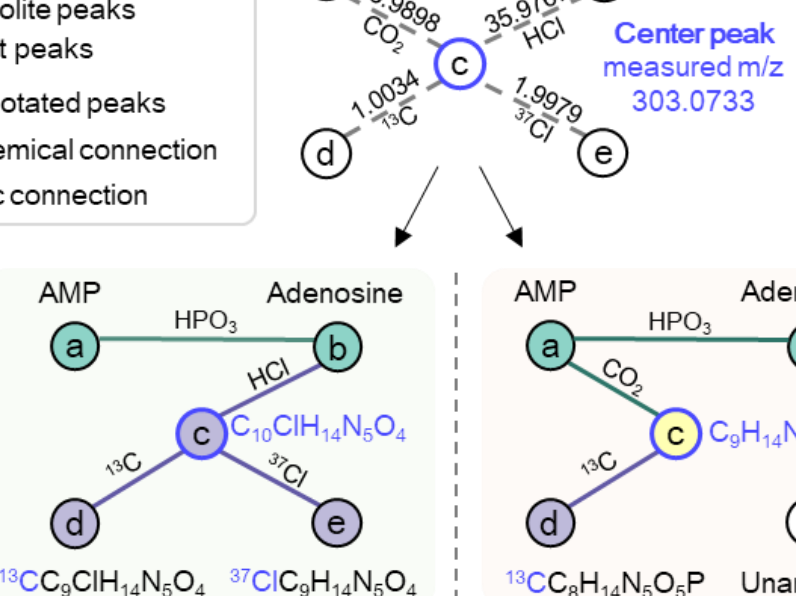

$\mathrm{C}_{10} \mathrm{H}_{13} \mathrm{~N}_{5} \mathrm{O}_{4}$ Adenosine

(b)

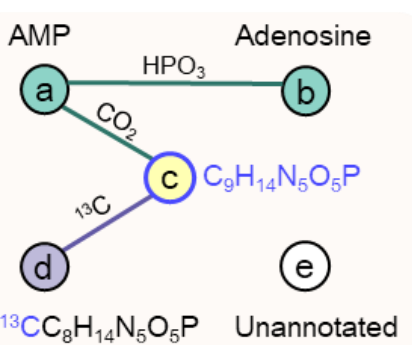

\begin{tabular}{|c|c|c|c|c|}
\hline Node & Annotation & Scoring criteria & Annotation & Scoring criteria \\
\hline$a$ & $\mathrm{C}_{10} \mathrm{H}_{14} \mathrm{~N}_{5} \mathrm{O}_{7} \mathrm{P}$ & $\mathrm{m} / \mathrm{z}, \mathrm{RT}, \mathrm{MS} 2$ & $\mathrm{C}_{10} \mathrm{H}_{14} \mathrm{~N}_{5} \mathrm{O}_{7} \mathrm{P}$ & $\mathrm{m} / \mathrm{z}, \mathrm{RT}, \mathrm{MS} 2$ \\
\hline$b$ & $\mathrm{C}_{10} \mathrm{H}_{13} \mathrm{~N}_{5} \mathrm{O}_{4}$ & $\mathrm{~m} / \mathrm{z}, \mathrm{RT}, \mathrm{MS} 2$ & $\mathrm{C}_{10} \mathrm{H}_{13} \mathrm{~N}_{5} \mathrm{O}_{4}$ & $\mathrm{~m} / \mathrm{z}, \mathrm{RT}, \mathrm{MS} 2$ \\
\hline c & $\mathrm{C}_{10} \mathrm{ClH}_{14} \mathrm{~N}_{5} \mathrm{O}_{4}$ & $\mathrm{~m} / \mathrm{z}$ & $\mathrm{C}_{9} \mathrm{H}_{14} \mathrm{~N}_{5} \mathrm{O}_{5} \mathrm{P}$ & $\mathrm{m} / \mathrm{z}$ \\
\hline$d$ & ${ }^{13} \mathrm{CC}_{9} \mathrm{ClH}_{14} \mathrm{~N}_{5} \mathrm{O}_{4}$ & $\mathrm{~m} / \mathrm{z}$ & ${ }^{13} \mathrm{CC}_{8} \mathrm{H}_{14} \mathrm{~N}_{5} \mathrm{O}_{5} \mathrm{P}$ & $\mathrm{m} / \mathrm{z}$ \\
\hline e & ${ }^{37} \mathrm{ClC}_{9} \mathrm{H}_{14} \mathrm{~N}_{5} \mathrm{O}_{4}$ & $\mathrm{~m} / \mathrm{z}$ & \multicolumn{2}{|c|}{ None } \\
\hline Summary & \multicolumn{2}{|c|}{5 good node scores } & 4 good nc & descores \\
\hline \multicolumn{5}{|l|}{ Edge } \\
\hline$a-b$ & $\mathrm{HPO}_{3}$ & MS2 similarity & $\mathrm{HPO}_{3}$ & MS2 similarity \\
\hline$a-c$ & \multicolumn{2}{|c|}{ None } & $\mathrm{CO}_{2}$ & MS2 similarity \\
\hline$b-c$ & $\mathrm{HCl}$ & Peak shape & \multicolumn{2}{|c|}{ None } \\
\hline$c-d$ & ${ }^{13} \mathrm{C}$ isotope & $\begin{array}{l}\text { Peak shape \& } \\
\text { Isotopic ratio }\end{array}$ & ${ }^{13} \mathrm{C}$ isotope & $\begin{array}{l}\text { Peak shape \& } \\
\text { Isotopic ratio }\end{array}$ \\
\hline$c-e$ & ${ }^{37} \mathrm{Cl}$ isotope & $\begin{array}{l}\text { Peak shape \& } \\
\text { Isotopic ratio }\end{array}$ & \multicolumn{2}{|c|}{ None } \\
\hline Summary & \multicolumn{2}{|c|}{4 good edge scores } & \multicolumn{2}{|c|}{3 good edge scores } \\
\hline Network & Total score & Optimal network & Total score & Optimal network \\
\hline & Higher & $\sqrt{ }$ & Lower & $x$ \\
\hline
\end{tabular}

B

\begin{tabular}{ccccc}
\hline & $\begin{array}{c}\text { Yeast } \\
\text { (Neg) }\end{array}$ & $\begin{array}{c}\text { Yeast } \\
\text { (Pos) }\end{array}$ & $\begin{array}{c}\text { Liver } \\
\text { (Neg) }\end{array}$ & $\begin{array}{c}\text { Liver } \\
\text { (Pos) }\end{array}$ \\
\hline $\begin{array}{c}\text { Total non- } \\
\text { background } \\
\text { peaks(nodes) }\end{array}$ & $\mathbf{5 5 8 8}$ & $\mathbf{9 8 3 3}$ & $\mathbf{8 1 9 1}$ & $\mathbf{1 2 1 2 8}$ \\
$\begin{array}{c}\text { Known } \\
\text { metabolite }\end{array}$ & 931 & 1140 & 1416 & 1636 \\
$\begin{array}{c}\text { Putative novel } \\
\text { metabolite }\end{array}$ & 686 & 1950 & 1122 & 2290 \\
$\begin{array}{c}\text { Artifact } \\
\text { Unannotated }\end{array}$ & 7225 & 5379 & 4568 & 6746 \\
\hline $\begin{array}{c}\text { Total } \\
\text { connections } \\
\text { (edges) }\end{array}$ & 9681 & 17019 & 15759 & $\mathbf{2 3 6 5 1}$ \\
$\begin{array}{c}\text { Biochemical } \\
\text { Abiotic }\end{array}$ & 5392 & 10173 & 9563 & 14934 \\
\hline
\end{tabular}

C
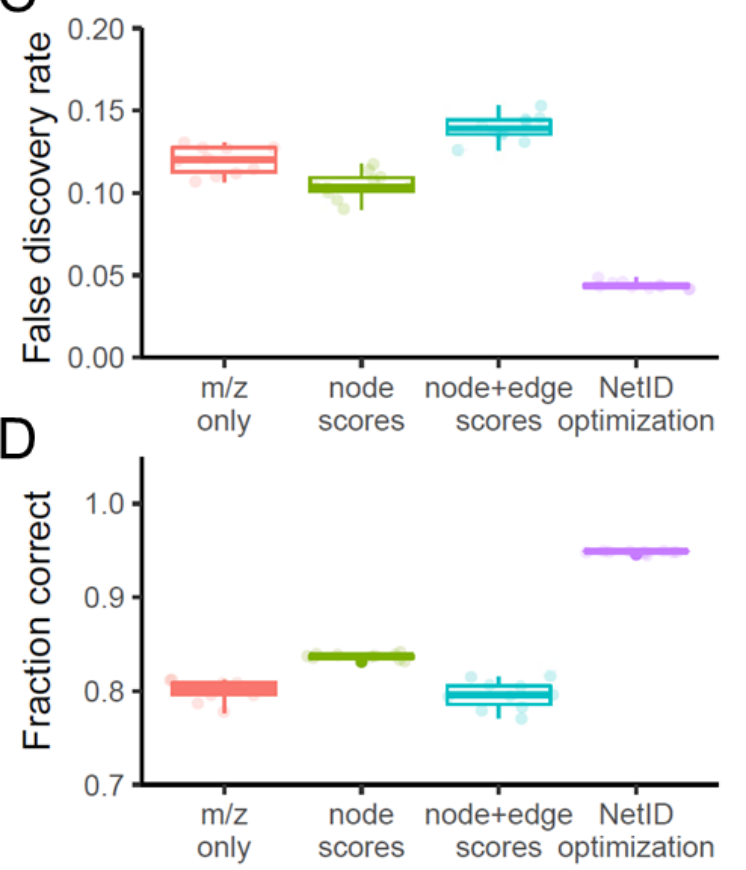

Figure 2. Utility of global network optimization. (A) An example network demonstrating the value of the global optimization step in NetID. Node $a$ and node $b$ match database formulae and are connected by an edge of phosphate $\left(\mathrm{HPO}_{3}\right)$. Node $c$ can be connected to either node $a$ or node $b$ through mutually incompatible annotations, resulting in two different candidate networks. The table below the two candidate networks shows the annotations and scoring criteria for each, with the left network preferred for more good node and edge annotations. (B) Summary table of NetID annotations of negative and positive mode LC-MS data from Baker's yeast and mouse liver. (C) False discovery rate estimated using target-decoy strategy. Each individual data point (circle) is from a different randomized decoy library ( $n=10$ randomized libraries were tested). (D) Fraction of 314 manually curated "ground truth" annotations made correctly (using the 10 libraries). Boxes show median and interquartile range. 

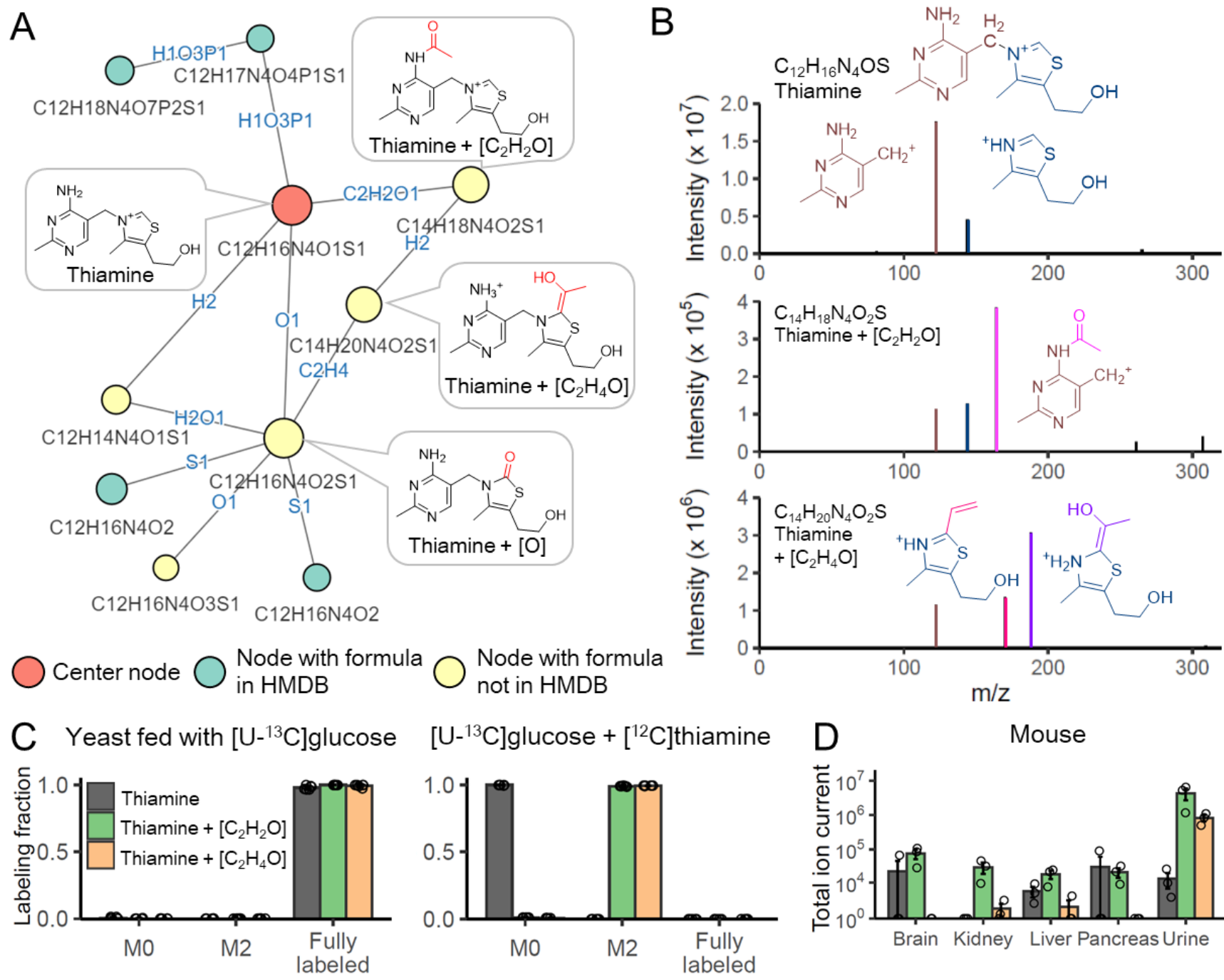

Center node $\bigcirc \begin{aligned} & \text { Node with formula } \\ & \text { in HMDB }\end{aligned} \underbrace{\text { Node with formula }}_{\text {not in HMDB }}$

$\left[\mathrm{U}-{ }^{13} \mathrm{C}\right]$ glucose $+\left[{ }^{12} \mathrm{C}\right]$ thiamine
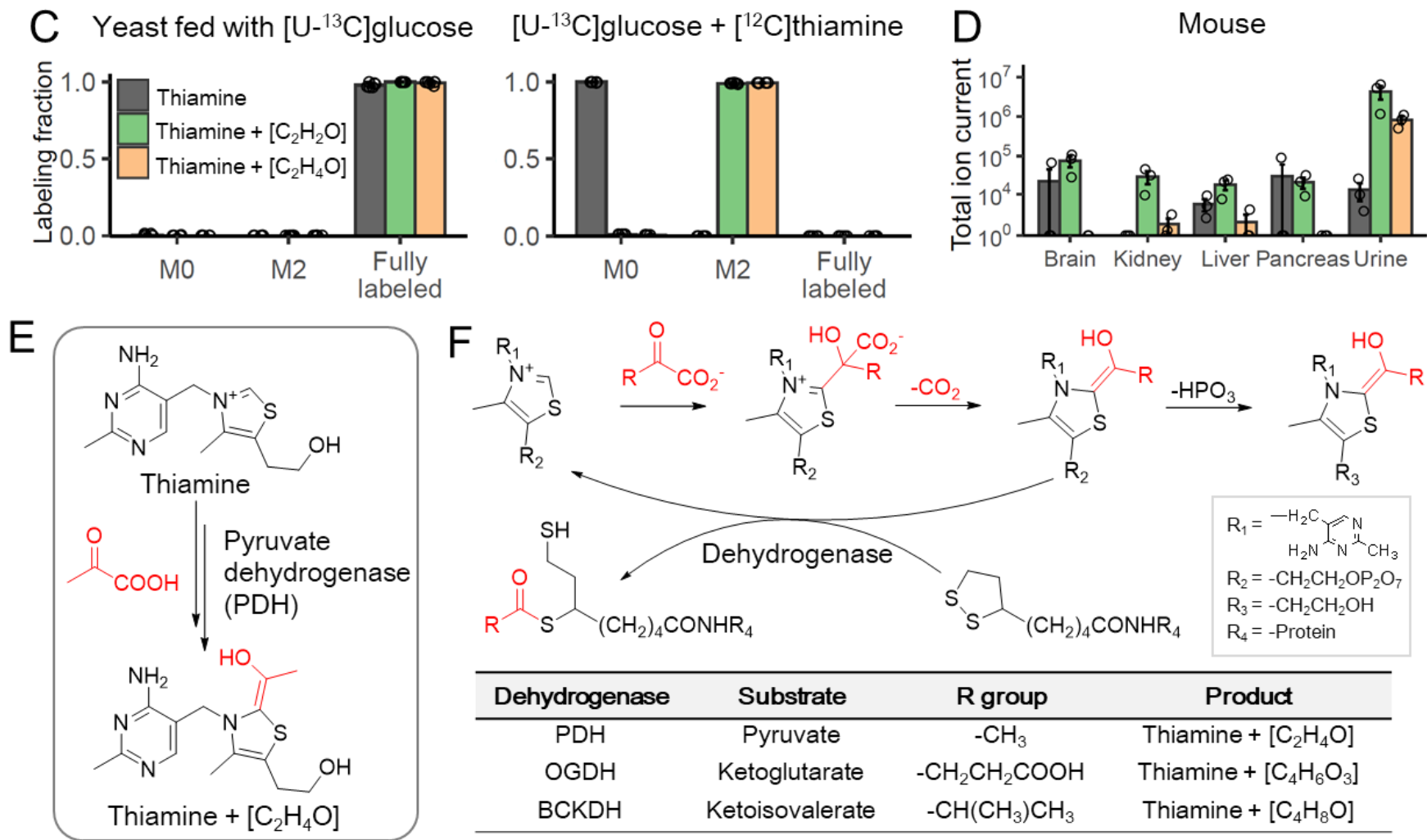

\begin{tabular}{cccc}
\hline Dehydrogenase & Substrate & R group & Product \\
\hline PDH & Pyruvate & $-\mathrm{CH}_{3}$ & Thiamine $+\left[\mathrm{C}_{2} \mathrm{H}_{4} \mathrm{O}\right]$ \\
OGDH & Ketoglutarate & $-\mathrm{CH}_{2} \mathrm{CH}_{2} \mathrm{COOH}$ & Thiamine $+\left[\mathrm{C}_{4} \mathrm{H}_{6} \mathrm{O}_{3}\right]$ \\
BCKDH & Ketoisovalerate & $-\mathrm{CH}\left(\mathrm{CH}_{3}\right) \mathrm{CH}_{3}$ & Thiamine $+\left[\mathrm{C}_{4} \mathrm{H}_{8} \mathrm{O}\right]$ \\
\hline
\end{tabular}

Figure 3. NetID reveals thiamine-derived metabolites in yeast. (A) Subnetwork surrounding thiamine. Nodes, connections, and formulae are direct output of NetID. Boxes with structures were manually added. (B) MS2 spectra of thiamine, thiamine $+\mathrm{C}_{2} \mathrm{H}_{2} \mathrm{O}$, and thiamine $+\mathrm{C}_{2} \mathrm{H}_{4} \mathrm{O}$, with proposed structures of the major fragments. (C) Labeling fraction of thiamine and its derivatives, in $\left[\mathrm{U}-{ }^{13} \mathrm{C}\right.$ ]glucose with and without unlabeled thiamine in the medium $(n=5)$. (D) The thiamine derivatives are also found in mouse tissues and urine $(n=3)$. (E) Proposed mechanism for formation of thiamine $+\mathrm{C}_{2} \mathrm{H}_{4} \mathrm{O}$. Pyruvate dehydrogenase (PDH) decarboxylates pyruvate, and adds the resulting $\left[\mathrm{C}_{2} \mathrm{H}_{4} \mathrm{O}\right]$ unit (in red) to thiamine. (F) The same enzymatic mechanism occurs in oxoglutarate dehydrogenase (OGDH) and branched-chain $\alpha$-ketoacid dehydrogenase complex (BCKDC), and generates thiamine $+\mathrm{C}_{4} \mathrm{H}_{6} \mathrm{O}_{3}$ and thiamine $+\mathrm{C}_{4} \mathrm{H}_{8} \mathrm{O}$ respectively. Bar represents mean values and error bar indicates s.d. in (C) and s.e. in (D). 


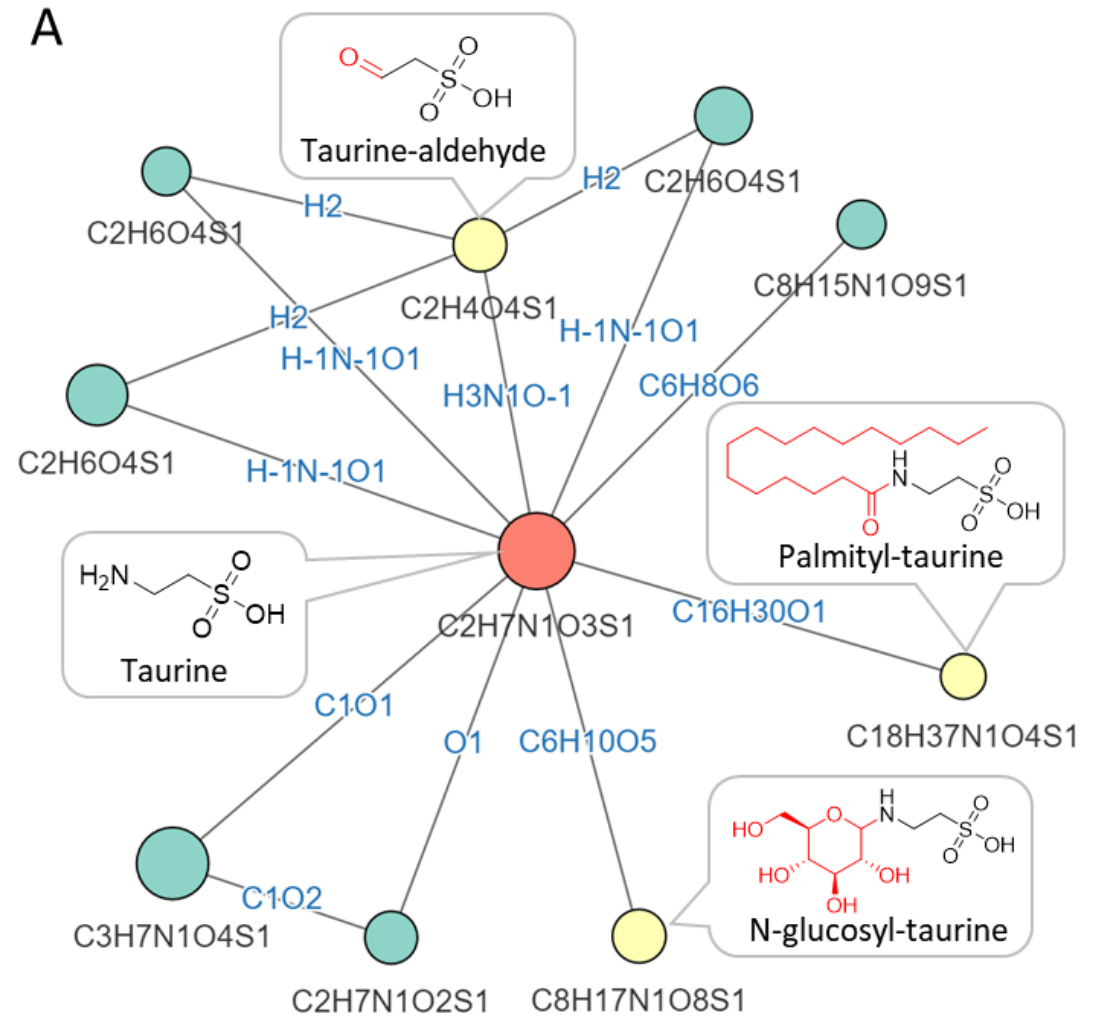

Center node
Node with formula not in HMDB
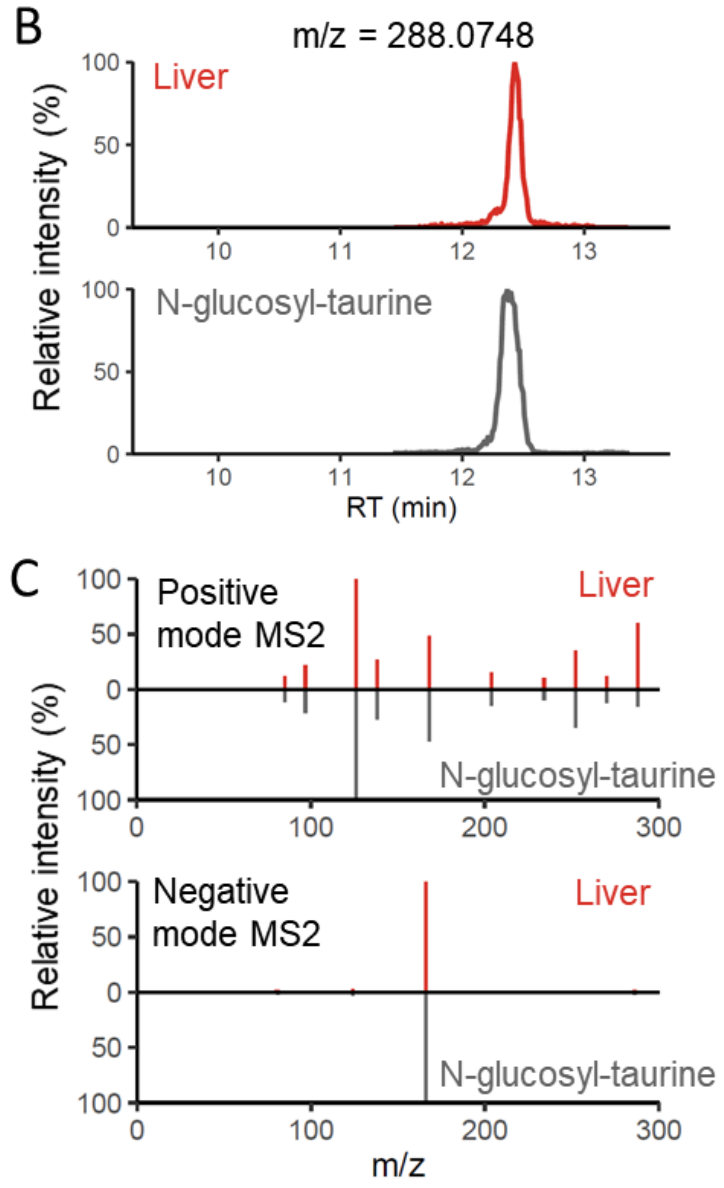

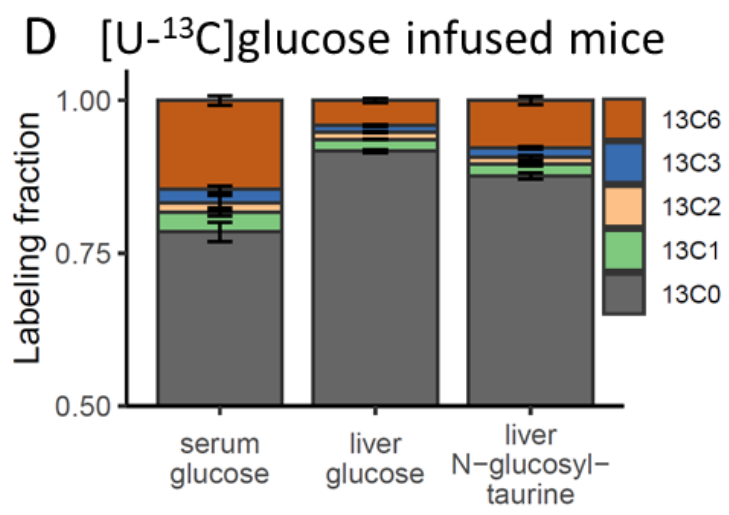

E N-glucosyl-taurine concentration in tissues

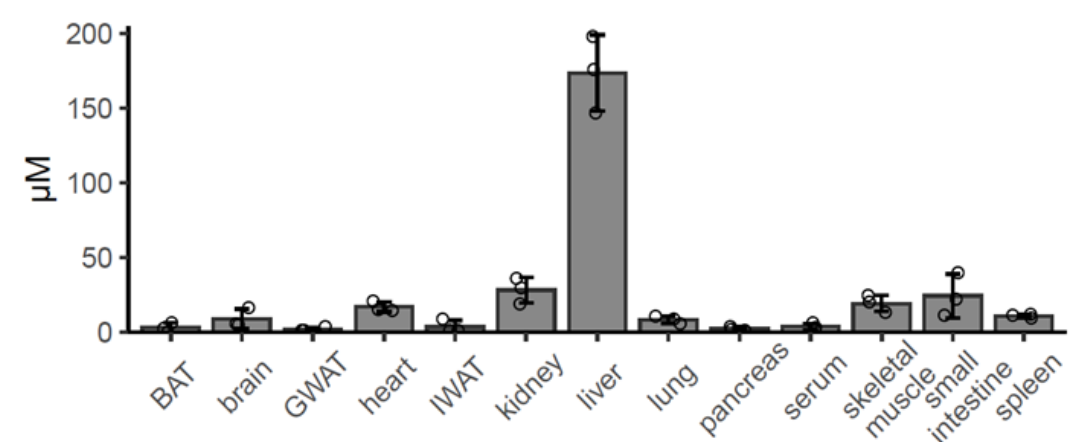

Figure 4. NetID discovers mammalian taurine derivatives. (A) Subnetwork surrounding taurine from mouse liver extract data. Nodes, connections, and formulae are direct output of NetID. Boxes with structures were manually added. (B) LC-MS chromatogram of N-glucosyl-taurine standard and the putative glucosyl-taurine from liver extract. (C) Top 10 abundant ion peaks in MS2 spectrum of glucosyl-taurine peak from liver extract (top), and synthetic N-glucosyl-taurine standard (bottom). (D) Isotope labeling pattern of putative glucosyl-taurine in mice, infused via jugular vein catheter for $2 \mathrm{~h}$ with $\left[\mathrm{U}-{ }^{13} \mathrm{C}\right] \mathrm{glucose}(\mathrm{n}=3)$. (E) Absolute $\mathrm{N}$-glucosyl-taurine concentration in murine serum and tissues $(n=3)$. Bar represents mean values and error bar indicates s.d. in (D) and s.e. in (E). 


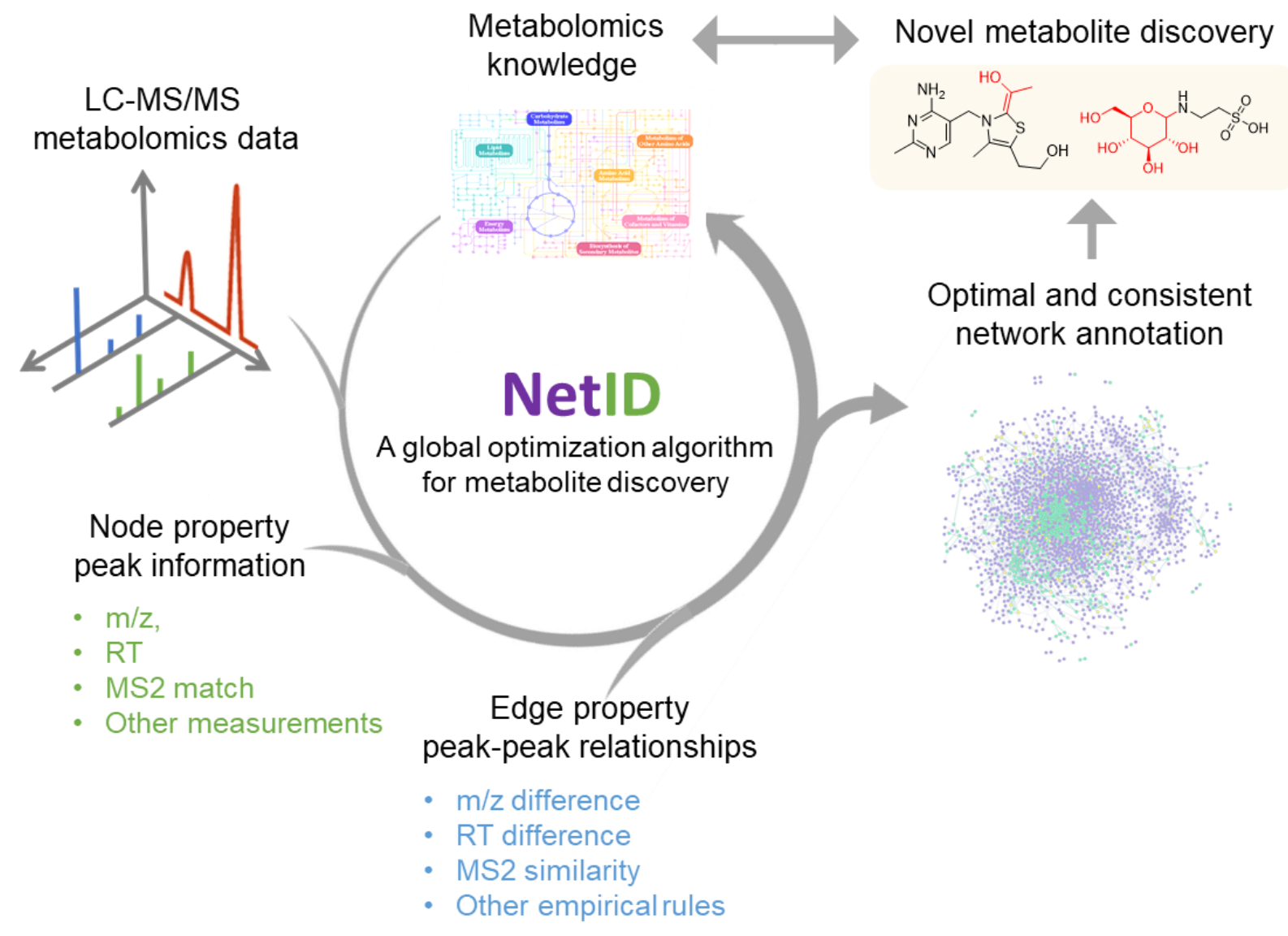

Figure 5. NetID applies global optimization for metabolomics data annotation and metabolite discovery. 